\title{
Clifford Operators and Riemann's Monodromy Problem
}

By

Tetsuji Miwa*

\section{$\S 1$. Introduction}

The purpose of this paper is to exploit the method of holonomic quantum fields developed in a series of papers [1], [2], [3] in solving Riemann's monodromy problem.

In [4] Wu, McCoy, Tracy and Barouch have shown that the scaled correlation functions $\tau_{-}(t)\left(T \uparrow T_{c}\right)$ and $\tau_{+}(t)\left(T \downarrow T_{c}\right)$ are expressible in a closed form using a solution $\eta(t)$ to the Painlevé equation of the third kind:

$$
\begin{aligned}
& \tau_{ \pm}(t)=\text { const. } t^{1 / 4}(1 \mp \eta(t)) \eta(t)^{-1 / 2} \\
& \times \exp \left(\int_{t}^{\infty} \frac{s\left\{\left(1-\eta(s)^{2}\right)^{2}-\eta^{\prime}(s)^{2}\right\}}{4 \eta(s)^{2}} d s\right), \\
& \eta^{\prime \prime}=\frac{1}{\eta}\left(\eta^{\prime}\right)^{2}-\frac{1}{t} \eta^{\prime}-\frac{1}{\eta}+\eta^{3} \text {. }
\end{aligned}
$$

Inspired by this Sato, Miwa and Jimbo revealed the unexpected link between the quantum field theory and the monodromy preserving deformation theory. Their method is roughly summarized as follows.

Step 1. The correlation function is expressed as the expectation value $\left\langle\varphi_{1} \cdots \varphi_{n}\right\rangle$ of a product $\varphi_{1} \cdots \varphi_{n}$ of Clifford operators $\varphi_{1}, \ldots, \varphi_{n}$. The special character of a Clifford operator is in the following form

$$
\begin{aligned}
& \varphi_{\mu}=: \exp \rho_{\mu}:, \\
& \rho_{\mu}=\iint d x d x^{\prime} R_{\mu}\left(x, x^{\prime}\right) \psi(x) \psi\left(x^{\prime}\right),
\end{aligned}
$$

where $\psi(x)$ is a free fermion field.

Step 2. Consider the wave function

Received October 8, 1980.

* Research Institute for Mathematical Sciences, Kyoto University Kyoto 606, Japan. 


$$
\left\langle\psi\left(x_{0}\right) \psi(x) \varphi_{1} \cdots \varphi_{n}\right\rangle \mid\left\langle\varphi_{1} \cdots \varphi_{n}\right\rangle .
$$

As a function of $x$ it enjoys a monodromy property independent of certain parameters $t_{1}, t_{2}, \ldots$ (which we call the deformation parameters) contained in $\varphi_{1}, \ldots, \varphi_{n}$.

Step 3. The monodromy property of the wave function leads us to a system of linear differential equation for the wave function with respect to $x$ and $t_{1}, t_{2}, \ldots$. The coefficients of this system are rational functions in $x$ whose coefficients in the partial fractions are unknown functions of the deformation parameters.

Step 4. As integrability conditions for this linear system we obtain a completely integrable system of non linear differential equations for the above unknown functions. We call it the deformation equation.

Step 5. Finally we can find an expression for the logarithmic derivative of the correlation function in terms of the unknown coefficients, which are now a solution to the deformation equation.

Using these techniques they found closed expressions for correlation functions in several physical models.

In [1] (Chapter II) they exploited this link in a converse way. They constructed a certain Clifford operator $\varphi(a ; L)$ depending on a point $a \in \boldsymbol{C}$ and an $m \times m$ matrix $L(|L| \ll 1)$ so that the wave function

$$
Y(x)_{\alpha \beta}=2 \pi i\left(x-x_{0}\right) \frac{\left\langle\psi_{\alpha}^{*}\left(x_{0}\right) \varphi\left(a_{1} ; L_{1}\right) \cdots \varphi\left(a_{n} ; L_{n}\right) \psi_{\beta}(x)\right\rangle}{\left\langle\varphi\left(a_{1} ; L_{1}\right) \cdots \varphi\left(a_{n} ; L_{n}\right)\right\rangle}
$$

enjoys the following monodromy property.

(R1) $Y(x)$ is multi-valued and holomorphic except at the branch points $a_{1}, \ldots, a_{n}$ and $\infty$.

(R2) $Y\left(x_{0}\right)=1$.

(R3) At $x=a_{\mu}$ we have

$$
Y(x)=\Phi_{\mu}(x)\left(x-a_{\mu}\right)^{L_{\mu}}
$$

where $\Phi_{\mu}(x)$ is locally holomorphic at $a_{\mu}$.

(R4) At $x=\infty$ we have

$$
Y(x)=\Phi_{\infty}(x)\left(\frac{1}{x}\right)^{L_{\infty}}
$$

where $\Phi_{\infty}(x)$ is locally holomorphic at $\infty$ and $L_{\infty}\left(\left|L_{\infty}\right| \ll 1\right)$ is determined by 


$$
e^{2 \pi i L_{1} \cdots} e^{2 \pi i L_{n}} e^{2 \pi i L_{00}}=1 .
$$

Steps 3 and 4 for this problem was solved by Schlesinger [5] a long time ago. If we take the normalization at $\infty$, Step 3 gives us the following linear system.

$$
\begin{aligned}
& \frac{\partial Y(x)}{\partial x}=\left(\sum_{\mu=1}^{n} \frac{A_{\mu}}{x-a_{\mu}}\right) Y(x), \\
& \frac{\partial Y(x)}{\partial a_{\mu}}=-\frac{A_{\mu}}{x-a_{\mu}} Y(x) .
\end{aligned}
$$

Then Step 4 gives us the Schlesinger's equation.

$$
d A_{\mu}=-\sum_{v \neq l l}\left[A_{\mu}, A_{v}\right] d \log \left(a_{\mu}-a_{v}\right),
$$

where $\quad d f=\sum_{\mu=1}^{n} \frac{\partial f}{\partial a_{\mu}} d a_{\mu}$.

Finally, Step 5 was completed as follows. For any solution $A_{\mu}$ to (1.11)

$$
\omega=\frac{1}{2} \operatorname{trace} \sum_{v \neq \mu} A_{\mu} A_{\nu} d \log \left(a_{\mu}-a_{v}\right)
$$

is a closed one form. If $A_{\mu}$ corresponds to the wave function $Y(x)$ in (1.5) we have

$$
\omega=d \log \left\langle\varphi\left(a_{1} ; L_{1}\right) \cdots \varphi\left(a_{n} ; L_{n}\right)\right\rangle .
$$

Recently, Ueno [6], [7] and Flaschka and Newell [8] initiated the study of monodromy preserving deformation of linear ordinary differential equation with irregular singularities. The general theory was established by Jimbo, Miwa and Ueno in [9]. Their work covers Steps 3 and 4. In particular, they derived the deformation equation and proved its complete integrability. Moreover they gave the definition of a closed one form $\omega$ and defined a special function $\tau$ by

$$
\omega=d \log \tau
$$

In Sections 2 and 3 of this paper we shall fill up Steps 1 and 2. Since we have already encountered irregular singularities in physical problems [2], [3], our task is just to compound general prescriptions. In Section 2, we shall give an infinite series expression for an $m \times m$ matrix $Y(x)$ with a prescribed data $a_{\mu}$, $t_{-j, \alpha}^{(\mu)}, \hat{\lambda}_{\alpha \beta}^{(\mu l)}$ and $C_{\alpha \beta}^{(\mu)}\left(\mu=1, \ldots, n ; j=0, \ldots, r_{\mu} ; l=1, \ldots, 2 r_{\mu} ; \alpha, \beta=1, \ldots, m\right)$. If $t_{-j, \alpha}^{(\mu)}$ 's and $\lambda_{\alpha \beta}^{\left(\mu_{l}\right)}$ 's are small, our series is convergent and gives us the solution to the monodromy problem. In Section 3, we construct a Clifford operator $\varphi_{\alpha}^{(\mu)}$ 
$(\mu=1, \ldots, n ; \alpha=1, \ldots, m)$ so that the infinite series in Section 2 is written in the following compact form

$$
Y(x)_{\alpha \beta}=2 \pi i\left(x-x_{0}\right) \frac{\left\langle\psi_{\alpha}^{*}\left(x_{0}\right) \varphi_{1}^{(1)} \cdots \varphi_{m}^{(n)} \psi_{\beta}(x)\right\rangle}{\left\langle\varphi_{1}^{(1)} \cdots \varphi_{m}^{(n)}\right\rangle} .
$$

Section 4 covers the difference scheme (Schlesinger transformation) of the monodromy preserving deformation theory, which was elaborated in [10], from the standpoint of Clifford operators. In particular, the reason why $\tau$ quotients [10] is expressed as determinants becomes clear. In Section 5 we prove that

$$
\omega=d \log \left\langle\varphi_{1}^{(1)} \cdots \varphi_{m}^{(n)}\right\rangle
$$

to fill up Step 5.

As an application of the method developed in this paper we shall prove the Painleve property of the deformation equation and the analyticity of the $\tau$ functions in a separate paper [11].

The results of this paper was announced in a short note [12].

The author would like to express his heartiest gratitude to M. Sato, K. Aomoto, K. Okamoto, M. Jimbo and K. Ueno for many helpful discussions.

\section{§2. Riemann's Monodromy Problem}

In this section we shall give an analytic expression for the solution $Y(x)$ $=\left(Y(x)_{\alpha \beta}\right)_{\alpha, \beta=1, \ldots, m}$ to the Riemann's monodromy problem. $Y(x)$ is given in the form

$$
\begin{aligned}
Y(x)_{\alpha \beta} & =\delta_{\alpha \beta}+2 \pi i\left(x-x_{0}\right) \sum_{\mu, v=1}^{n} \sum_{\alpha^{\prime}, \beta^{\prime}=1}^{m} \int_{I_{\varepsilon}^{(\mu)}} d y \\
& \times\left(C^{(\mu)-1}\right)_{\alpha \alpha^{\prime}} \frac{1}{2 \pi} \frac{i}{x_{0}-y} R_{\alpha^{\prime} \beta^{\prime}}^{(\mu \nu)}(y, x) C_{\beta^{\prime} \beta}^{(\nu)}
\end{aligned}
$$

where

$$
\begin{aligned}
R_{\alpha^{\prime} \beta^{\prime}}^{(\mu \nu)}(y, x) & =\sum_{j=0}^{\infty} \sum_{v_{1}, \ldots, v_{j-1}=1}^{n} \sum_{\alpha_{1}, \ldots, \alpha_{j-1}=1}^{m} \int d x_{1} \cdots \int d x_{j} \\
& \times R_{\alpha^{\prime}}^{(\mu)}\left(y, x_{1}\right) K_{\alpha^{\prime} \alpha_{1}}^{\left(\mu v_{1}\right)} \cdots R_{\alpha_{j-1}}^{\left(v_{j-1}\right)}\left(x_{j-1}, x_{j}\right) K_{\alpha_{j-1} \beta^{\prime}}^{\left(v_{j-1} v\right)} R_{\beta^{\prime}}^{(v)}\left(x_{j}, x\right) \\
& =\delta_{\mu v} \delta_{\alpha^{\prime} \beta^{\prime}} R_{\alpha^{\prime}}^{(\mu)}(y, x)+\int d x_{1} R_{\alpha^{\prime}}^{(\mu)}\left(y, x_{1}\right) K_{\alpha^{\prime} \beta^{\prime}}^{(\mu v)} R_{\beta^{\prime}}^{(v)}\left(x_{1}, x\right)+\cdots
\end{aligned}
$$

We shall take the following steps. 
i) We state the monodromy properties (M1)-(M6) which characterize $Y(x)$.

ii) We prove the convergence of the infinite series for $R_{\alpha^{\prime} \beta^{\prime}}^{(\mu \nu)}(y, x)$.

iii) We show that $Y(x)$ enjoys the prescribed monodromy property.

Let $a_{1}, \ldots, a_{n}$ be points in $\mathbb{C}$. We assume that $\operatorname{Im} a_{1}>\cdots>\operatorname{Im} a_{n}$. We denote by $\Gamma_{v}$, the half line $\left\{x \in \mathbb{C} \mid \operatorname{Im}\left(x-a_{v}\right)=0, \operatorname{Re}\left(x-a_{v}\right) \leqq 0\right\}$.

(M1) $Y(x)$ is an $m \times m$ matrix holomorphic in $\boldsymbol{C}-\cup_{v=1}^{n} \Gamma_{v}$.

We fix a normalization point $x_{0} \in \mathbb{C}-\cup_{v=1}^{n} \Gamma_{v}$ for $Y(x)$.

(M2) $Y\left(x_{0}\right)=1$.

Let $M^{(1)}, \ldots, M^{(n)}$ be $m \times m$ invertible matrices. We assume that

$$
M^{(n)} \cdots M^{(1)}=1 .
$$

(M3) $Y(x)$ can be continued analytically across $\Gamma_{v}-\left\{a_{v}\right\}$ from both sides and the boundary values $Y(x \pm i 0)\left(x \in \Gamma_{v}-\left\{a_{v}\right\}\right)$ satisfy

$$
Y(x+i 0)=Y(x-i 0) M^{(v)} .
$$

$M^{(v)}$ is called the monodromy matrix at $a_{v}$.

For each $v$ we choose a set of $m \times m$ matrices which we call the monodromy data at $a_{v}: T_{-r_{v}}^{(v)}, \ldots, T_{-1}^{(v)}, T_{0}^{(v)}, \Lambda_{1}^{(v)}, \ldots, \Lambda_{2 r_{v}}^{(v)}, C^{(v)}$. The non negative integer $r_{v}$ is called the rank of irregularity of $Y(x)$ at $a_{v}$. We choose $T_{-j}^{(v)}=$ $\left(t_{-j, \alpha}^{(v)} \delta_{\alpha \beta}\right)_{\alpha, \beta=1, \ldots, m}$ to be diagonal and set

$$
e_{\alpha}^{(v)}(x)=\exp \left(\sum_{j=1}^{r_{v}} t_{-j, \alpha}^{(v)} \frac{\left(x-a_{v}\right)^{-j}}{-j}+t_{0, \alpha}^{(v)} \log \left(x-a_{v}\right)\right) .
$$

If $r_{v}=0$ we choose $T_{0}^{(v)}$ and $C^{(v)}$ so that $C^{(v)}$ is invertible and

$$
C^{(v)-1} e^{2 \pi i T_{0}^{(v)}} C^{(v)}=M^{(v)} .
$$

(M4) If $r_{v}=0,\left(Y(x) C^{(v)-1}\right)_{\alpha \beta} e_{\beta}^{(v)}(x)^{-1}$ is holomorphic at $a_{v}$. If $r_{v} \geqq 1$ we define sectors at $a_{v}$ :

$$
\begin{array}{r}
\mathscr{S}_{l, \delta}^{(v)}=\left\{x \in \mathbb{C}|0<| x-a_{v} \mid<\delta, \pi \frac{l-1}{r_{v}}-\delta<\arg \left(x-a_{v}\right)<\pi \frac{l}{r_{v}}\right\}, \\
\left(\delta>0, l=1, \ldots, 2 r_{v}+1\right) .
\end{array}
$$

We assume that $t_{-r_{v}, \alpha}^{(v)} \neq t_{-r_{v}, \beta}^{(v)}$ for $\alpha \neq \beta$, and choose sufficiently small $\delta$ so that

$$
\operatorname{Re}_{\alpha \beta}^{(v)}(x) \underset{\text { def }}{=\operatorname{Re}}\left(t_{-r_{v}, \alpha}^{(v)} \frac{\left(x-a_{v}\right)^{-r_{v}}}{-r_{v}}-t_{-r_{v}, \beta}^{(v)} \frac{\left(x-a_{v}\right)^{-r_{v}}}{-r_{v}}\right) \neq 0
$$

for $x \in \mathscr{S}_{l, \delta}^{(v)} \cap \mathscr{S}_{l+1, \delta}^{(v)}$. The monodromy data $\Lambda_{l}^{(v)}=\left(\lambda_{\alpha \beta}^{(v l)}\right)_{\alpha, \beta=1, \ldots, m}$ is a nilpotent matrix such that 


$$
\lambda_{\alpha \beta}^{(v l)}=0 \quad \text { if } \quad \operatorname{Re}_{\alpha \beta}^{(v)}(x) \geqq 0 \quad \text { for } \quad x \in \mathscr{S}_{l, \delta}^{(v)} \cap \mathscr{S}_{l+1, \delta}^{(v)} .
$$

We set

$$
S_{l}^{(v)}=\left(1+\Lambda_{l}^{(v)}\right)^{-1}
$$

and require that

$$
C^{(v)-1} e^{2 \pi i T_{0}^{(v)}} S_{2 r_{v}}^{(v)-1} \cdots S_{1}^{(v)-1} C^{(v)}=M^{(v)} .
$$

(M5) If $r_{v}>1,\left(Y(x) C^{(v)-1} S_{1}^{(v)} \ldots S_{l-1}^{(v)}\right)_{\alpha \beta} e_{\beta}^{(v)}(x)^{-1}\left(l=1, \ldots, 2 r_{v}+1\right)$ has an asymptotic expansion in the sector $\mathscr{S}_{l, \delta}^{(v)}$, which is independent of $l$.

(M6) $Y(x)$ is holomorphic at $x=\infty$.

From (2.3), (2.6) and (2.11) we have $\exp 2 \pi i\left(\sum_{v=1}^{n} \sum_{\alpha=1}^{m} t_{0, \alpha}^{(v)}\right)=1$. We choose $t_{0, \alpha}^{(v)}$ so that

$$
\sum_{v=1}^{n} \sum_{\alpha=1}^{m} t_{0, \alpha}^{(v)}=0
$$

Then we have

Proposition 2.1. If an $m \times m$ matrix $Y(x)$ satisfying (M1)-(M6) ever exists, it is unique. Moreover we have

$$
\operatorname{det} Y(x)=\prod_{v=1}^{n} \prod_{\alpha=1}^{m} e_{\alpha}^{(v)}(x) / e_{\alpha}^{(v)}\left(x_{0}\right)
$$

Proof. (M1), (M3), (M4) and (M5) imply that $\operatorname{det} Y(x) \prod_{v=1}^{n} \prod_{\alpha=1}^{m}$. $e_{\alpha}^{(v)}(x)^{-1}$ is holomorphic everywhere in $\mathbb{C}$. (M6) and (2.12) imply that it is holomorphic at $\infty$, hence it is constant. The constant is determined by (M2) and we obtain (2.13). Let $Y_{1}(x)$ and $Y_{2}(x)$ be two matrices satisfying (M1)(M6). Since $Y_{1}(x)$ and $Y_{2}(x)$ satisfy (M3), $Y_{1}(x) Y_{2}(x)^{-1}$ is single-valued. (M4)(M5) and (2.13) imply that it is holomorphic everywhere in $\mathbb{P}^{1}$, hence it is constant. Then (M2) implies $Y_{1}(x) Y_{2}(x)^{-1}=1$.

In (2.2) we set

$$
\begin{aligned}
& R_{\alpha}^{(\mu)}\left(x, x^{\prime}\right)=\frac{1}{2 \pi i\left(x-x^{\prime}\right)} \frac{e_{\alpha}^{(\mu)}\left(x^{\prime}\right)}{e_{\alpha}^{(\mu)}(x)}, \\
& K_{\alpha \beta}^{(\mu \nu)}= \begin{cases}\left(C^{(\mu)} C^{(v)-1}\right)_{\alpha \beta} & \mu \neq v, \\
\lambda_{\alpha \beta}^{(v l)} & \mu=v, \alpha \neq \beta, \\
0 & \mu=v, \alpha=\beta .\end{cases}
\end{aligned}
$$

The precise meaning of the second line of (2.15) will be explained below (see (2.17)). 
Now we shall specify the contour for the $x_{k}$-integration $(k=1, \ldots, j)$ in (2.2). We set

$$
\begin{aligned}
& I_{\varepsilon}^{(v)}=I_{\varepsilon-}^{(v)} \cup I_{\varepsilon^{0}}^{(v)} \cup I_{\varepsilon+}^{(v)}, \\
& I_{\varepsilon \pm}^{(v)}=\left\{x \in \mathbb{C} \mid \operatorname{Im}\left(x-a_{v}\right)= \pm \varepsilon, \operatorname{Re}\left(x-a_{v}\right) \leqq 0\right\}, \\
& I_{\varepsilon^{0}}^{(v)}=\left\{x \in \mathbb{C}|| x-a_{v} \mid=\varepsilon,-\frac{\pi}{2} \leqq \arg \left(x-a_{v}\right) \leqq \frac{\pi}{2}\right\} .
\end{aligned}
$$

If $v_{k-1} \neq v_{k}$, the contour for $x_{k}$-integration is $I_{\varepsilon}^{\left(v_{k}\right)}$ in anticlockwise direction. We set

$$
\begin{aligned}
& I I_{\varepsilon}^{(v l)}=I I_{\varepsilon 1}^{(v l)} \cup I_{\varepsilon 2}^{(v l)}, \\
& I I_{\varepsilon 1}^{(v l)}=\left\{x \in \mathbb{C} \mid \operatorname{Im}\left(x-a_{v}\right)=\frac{\varepsilon}{2}-\frac{l-1}{2 r_{v}-1}, \operatorname{Re}\left(x-a_{v}\right) \leqq-\varepsilon\right\} .
\end{aligned}
$$

$I I_{\varepsilon_{2}}^{(v l)}$ connects $a_{v}-\varepsilon+i\left(\frac{1}{2}-\frac{l-1}{2 r_{v}-1}\right) \varepsilon$ with $a_{v}$ so that $I I_{\varepsilon}^{(v, l)}$ is a $C^{2}$-curve and tangential to the line $\left\{x \in \mathbb{C} \mid \arg \left(x-a_{v}\right)=\frac{\pi}{r} l-\frac{\delta}{2}\right\}$. We choose $I I_{\varepsilon 2}^{(v l)}$ $\left(l=1, \ldots, 2 r_{v}\right)$ so that they are inside of $I_{\varepsilon}^{(v)}$ and have no intersection. If $v_{k-1}$ $=v_{k}$ the contour for $x_{k}$-integration is the union $\bigcup_{l=1}^{2 r_{v}} I I_{\varepsilon}^{\left(v_{k} l\right)}$, where the direction of contour is from $-\infty$ to $a_{v}$. For $x_{k} \in I I_{\varepsilon}^{\left(v_{k} l\right)}$ we set

$$
K_{\alpha_{k-1} \alpha_{k}}^{\left(v_{k}-1 v_{k}\right)}=\lambda_{\alpha_{k-1}\left(v_{k} l\right)}^{\left(\alpha_{k}\right.}
$$

Note that $\lambda_{\alpha \beta}^{(\nu l)} \neq 0$ only if $I I_{\varepsilon}^{(v l)}$ goes into $a_{v}$ through a decreasing sector for $e_{\alpha}^{(v)}(x) / e_{\beta}^{(v)}(x)$. We say that the $x_{k}$-integration is type I (resp. II) if $v_{k-1} \neq v_{k}$ (resp. $v_{k-1}=v_{k}$ ). If the contours for $x_{k-1}$ and $x_{k}$ are both $I I_{\varepsilon}^{(\mu l)}$, we choose $x_{k}$ on the right bank of the contour for $x_{k-1}$.

Under the above prescription for the contours we have

Proposition 2.2. The infinite series (2.2) is convergent for sufficiently small values of parameters $t_{-j, \alpha}^{(v)}$ and $\lambda_{\alpha \beta}^{(v)}\left(v=1, \ldots, n ; j=0,1, \ldots, r_{v} ; \alpha, \beta=1, \ldots, m\right.$; $\left.l=1, \ldots, 2 r_{v}\right)$.

Proof. We follow essentially the argument in Section 2.3 in [1]. We set $\tilde{e}_{\alpha}^{(v)}(x)=\exp \left(\sum_{j=1}^{r_{v}} t_{-j, \alpha}^{(v)} \frac{\left(x-a_{v}\right)^{-j}}{-j}\right)$. Since $t_{0 \alpha}^{(k)}$ 's are small and $\lambda_{\alpha \beta}^{(v l)}$ 's are zero in increasing sectors, $R_{\alpha^{\prime}}^{(\mu)}\left(y, x_{1}\right) \tilde{e}_{\alpha^{\prime}}^{(\mu)}\left(x_{1}\right)^{-1}$ (resp. $\tilde{e}_{\alpha_{j-1}}^{\left(v_{j-1}\right)}\left(x_{j}\right) R_{\beta^{\prime}}^{(\nu)}\left(x_{j}, x\right)$ ) is squareintegrable in $x_{1}$ (resp. $x_{j}$ ) on its contour of the integration. Moreover the kernel $\tilde{e}_{\alpha_{k-1}}^{\left(v_{k-1}\right)}\left(x_{k}\right) R_{\alpha_{k}}^{\left(v_{k}\right)}\left(x_{k}, x_{k+1}\right) \tilde{e}_{\alpha_{k}}^{\left(v_{k}\right)}\left(x_{k+1}\right)^{-1}$ defines a bounded linear integral operator from the space of square-integrable functions on the contour of $x_{k+1}$ to that of $x_{k}$ (see Propositions 2.3.2 and 2.3.8 in [1]). Hence the integral 


$$
\int d x_{1} \cdots \int d x_{j} R_{\alpha^{\prime}}^{(\mu)}\left(y, x_{1}\right) K_{\alpha^{\prime} \alpha_{1}}^{\left(\mu \nu_{1}\right)} \cdots R_{\beta^{\prime}}^{(y)}\left(x_{j}, x\right)
$$

is convergent. The frequency of the case where the $x_{k}$-integration is of type I and the $x_{k+1}$-integration is of type II does not exceed one half among $k=1, \ldots$, $j-1$. If $x_{k}$-integration is of type II, we can take $K_{\alpha_{k}-1 \alpha_{k}}^{\left(v_{k}-1 v_{k}\right)}=\lambda_{\alpha_{k}-1 \alpha_{k}}^{\left(v_{k} l\right)}$ as small as we like. Thus in order to prove the convergence of (2.2) it is sufficient to show that the operator norm of the operator with the kernel $\tilde{e}_{\alpha_{k-1}}^{\left(v_{k-1}\right)}\left(x_{k}\right) R_{\alpha_{k}}^{\left(v_{k}\right)}\left(x_{k}, x_{k+1}\right)$. $\tilde{e}_{\alpha_{k}}^{\left(v_{k}\right)}\left(x_{k+1}\right)^{-1}$, where both the $x_{k}$ and $x_{k+1}$-integrations are of type I, can bc diminished arbitrarily by choosing $t_{0, \alpha_{k}}^{\left(v_{k}\right)}, \ldots, t_{r_{v_{k}}, \alpha_{k}}^{\left(v_{v^{\prime}}\right)}$ to be small. We modify the contour $I^{\left(v_{k}\right)}$ for $x_{k}$ to the following.

$$
\begin{aligned}
& \tilde{I}_{\varepsilon}^{\left(v_{k}\right)}=\tilde{I}_{ \pm \varepsilon}^{\left(v_{k}\right)} \cup \tilde{I}_{0 \varepsilon}^{\left(v_{k}\right)}, \\
& \tilde{I}_{ \pm \varepsilon}^{\left(v_{k}\right)}=\left\{x \in C \mid \operatorname{Im}\left(x-a_{v_{k}}\right)=0, \quad \operatorname{Re}\left(x-a_{v_{k}}\right) \leqq-\varepsilon\right\}, \\
& \tilde{I}_{0 \varepsilon}^{\left(v_{k}\right)}=\left\{x \in C|| x-a_{v_{k}} \mid=\varepsilon\right\} .
\end{aligned}
$$

On $\tilde{I}_{ \pm \varepsilon}^{\left(v_{k}\right)}$ we have

$$
\begin{aligned}
& R_{x_{k}}^{\left(v_{k}\right)}\left(x_{k}-i 0, x_{k+1}\right)-R_{\alpha_{k}}^{\left(v_{k}\right)}\left(x_{k}+i 0, x_{k+1}\right) \\
& \quad=-2 i \sin \pi t_{0, \alpha_{k}}^{\left(v_{k}\right)}\left|x_{k}-a_{v_{k}}\right|^{t\left(v_{\alpha_{k}}\right)} \\
& \quad \times \exp \left(\sum_{j=1}^{r_{k}} t_{-j, \alpha_{k}}^{\left(v_{k_{k}}\right)} \frac{\left(x_{k}-a_{v_{k}}\right)^{-j}}{-j}\right) \frac{e_{\alpha_{k}}^{\left(v_{k}\right)}\left(x_{k+1}\right)}{2 \pi i\left(x_{k}-x_{k+1}\right)} .
\end{aligned}
$$

Thus we have a small factor $\sin \pi t_{0, \alpha_{k}}^{\left(v_{k}\right)}$. As for the contour $\tilde{I}_{0 \varepsilon}^{\left(v_{k}\right)}$ we can diminish the operator norm by choosing $\varepsilon$ and $t_{-j, \alpha_{k}}^{\left(v_{k}\right)}\left(j=0,1, \ldots, r_{v_{k}}\right)$ to be small.

Now we consider (2.1). The contour for $y$ is one of $I_{\varepsilon}^{(1)}, \ldots, I_{\varepsilon}^{(n)}$. They divide $\boldsymbol{C}$ into $n+1$ regions. We take $x_{0}$ to be outside of all these contours. The contour for $x_{j}$ may be any of $I_{\varepsilon}^{(v)}, I I_{\varepsilon}^{(v l)}\left(v=1, \ldots, n ; l=1, \ldots, 2 r_{v}\right)$ which divide $\boldsymbol{C}$ into $2 \sum_{v=1}^{n} r_{v}+1$ regions. We denote them by $\mathscr{R}_{0}$ and $\mathscr{R}^{(v l)}(v=1, \ldots$, $\left.n ; l=1, \ldots, 2 r_{v}\right) . \mathscr{R}^{(v l)}$ is the region which contains the segment $\{x \in C \mid 0$ $\left.<\left|x-a_{v}\right| \ll 1, \arg \left(x-a_{v}\right)=\pi(l-1 / 2) / r_{v}\right\}$. In $(2.1)$ we take $x$ to be in $\mathscr{R}_{0}$. Then we have

Theorem 2.3. For sufficiently small values of parameters $t_{-j, \alpha}^{(\mu)}$ and $\lambda_{\alpha \beta}^{\left(\mu_{l}\right)}$ $\left(\mu=1, \ldots, n ; j=0,1, \ldots, r_{\mu} ; \alpha, \beta=1, \ldots, m ; l=1, \ldots, 2 r_{\mu}\right)$ the $m \times m$ matrix $Y(x)$ given by (2.1) satisfies (M1)-(M6). At $x=a_{v}\left(Y(x) C^{(v)-1} S_{1}^{(v)} \cdots S_{l-1}^{(v)}\right)_{\alpha \beta} e_{\beta}^{(v)}(x)^{-1}$ has an asymptotic expansion of the form

$$
\sum_{v_{0}=1}^{n} \sum_{\alpha_{0}=1}^{m} \int_{I_{\varepsilon}^{\left(v_{0}\right)}} d y\left(C^{\left(v_{0}\right)-1}\right)_{\alpha_{\alpha_{0}}} \frac{1}{2 \pi} \frac{i}{x_{0}-y}
$$




$$
\begin{aligned}
& \times \sum_{j=0}^{\infty} \sum_{v_{1}, \ldots, v_{j-1}=1}^{n} \sum_{\alpha_{1}, \ldots, \alpha_{j-1}=1}^{m} \int d x_{1} \cdots \int d x_{j} \\
& \times R_{\alpha_{0}}^{\left(v_{0}\right)}\left(y, x_{1}\right) K_{\alpha_{0} \alpha_{1}}^{\left(v_{0} v_{1}\right)} \cdots R_{\alpha_{j}-1}^{\left(v_{j}\right)}\left(x_{j-1}, x_{j}\right) K_{\alpha_{j-1}}^{\left(v_{j-1} v\right)} \\
& \times e_{\beta}^{(v)}\left(x_{j}\right)^{-1}\left(\frac{a_{v}-x_{0}}{x_{j}-a_{v}}+\sum_{k=1}^{\infty} \frac{x_{j}-x_{0}}{\left(x_{j}-a_{v}\right)^{k+1}}\left(x-a_{v}\right)^{k}\right) .
\end{aligned}
$$

Proof. Let us consider the local behavior of $Y(x)$ at $x=a_{v}$. We divide $Y(x) C^{(v)-1}$ into two parts: $Y(x) C^{(v)-1}=F_{1}^{(v)}(x)+F_{2}^{(v)}(x)$ where

$$
\begin{gathered}
F_{1}^{(v)}(x)_{\alpha \beta}=\left(C^{(v)-1}\right)_{\alpha \beta}+2 \pi i\left(x-x_{0}\right) \sum_{\substack{\mu, \alpha=1 \\
\kappa \neq \nu}}^{n} \sum_{\alpha^{\prime}, \beta^{\prime}=1}^{m} \int_{I_{\varepsilon}^{(\mu)}} d y \\
\times\left(C^{(\mu)-1}\right)_{\alpha \cdot \alpha^{\prime}} \frac{1}{2 \pi} \frac{i}{x_{0}-y} R_{\alpha^{\prime} \beta^{\prime}}^{\left(\mu_{\kappa}\right)}(y, x)\left(C^{(\kappa)} C^{(v)-1}\right)_{\beta^{\prime} \beta}, \\
F_{2}^{(v)}(x)_{\alpha \beta}=2 \pi i\left(x-x_{0}\right) \sum_{\mu=1}^{n} \sum_{\alpha^{\prime}=1}^{m} \int_{I_{\varepsilon}^{(\mu)}} d y \\
\times\left(C^{(\mu)-1}\right)_{\alpha \cdot \alpha^{\prime}} \frac{1}{2 \pi} \frac{i}{x_{0}-y} R_{\alpha^{\prime} \beta}^{(\mu \nu)}(y, x) .
\end{gathered}
$$

When we consider $F_{s}^{(v)}(x)(s=1,2, \ldots)$ with $x$ in $R^{(v l)}$, we denote it by $F_{s}^{(v l)}(x)$. It is obvious that $F_{1}^{(v)}(x)=F_{1}^{(v l)}(x)$.

Lemma 2.4. $Y(x) C^{(v)-1}$ can be continued analytically into $\mathscr{R}^{(v 1)}$ across $I_{\varepsilon}^{(v)}$ and satisfies

$$
Y(x) C^{(v)-1}=F_{2}^{(v 1)}(x) .
$$

Proof. We divide $F_{2}^{(v)}(x)$ into two parts: $F_{2}^{(v)}(x)=F_{3}^{(v)}(x)+F_{4}^{(v)}(x)$ where

$$
\begin{aligned}
& F_{4}^{(v)}(x)_{\alpha \beta}=2 \pi i\left(x-x_{0}\right) \sum_{\mu=1}^{n} \sum_{\alpha^{\prime}=1}^{m} \int_{I_{\varepsilon}}^{(\mu)} d y \\
& \times\left(C^{(\mu)-1}\right)_{\alpha \alpha^{\prime}} \frac{1}{2 \pi} \frac{i}{x_{0}-y}\left\{\delta_{\mu \nu} \delta_{\alpha^{\prime} \beta} R_{\beta}^{(v)}(y, x)\right. \\
& +\sum_{j=1}^{\infty} \sum_{\substack{v_{1}, \ldots, v_{j}=1 \\
v_{j}-1 \neq v}}^{n} \sum_{\alpha_{1}, \ldots, \alpha_{j-1}=1}^{m} \int d x_{1} \ldots \int d x_{j}
\end{aligned}
$$

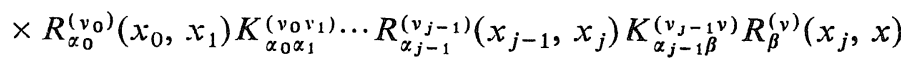

$$
\begin{aligned}
& \left(v_{0}=\mu, \alpha_{0}=\alpha^{\prime}, x_{0}=y\right) \text {. }
\end{aligned}
$$

Since the $x_{j}$-integration in $F_{3}^{(v)}(x)$ is of type II, the analytic continuation of $F_{3}^{(v)}(x)$ into $\mathscr{R}^{(v 1)}$ across $I_{\varepsilon}^{(v)}$ coincides with $F_{3}^{(v 1)}(x)$. The contour for $x_{j}$ in $F_{4}^{(v)}(x)$ is $I_{\varepsilon}^{(v)}$. The analytic continuation of $F_{4}^{(v)}(x)$ into $\mathscr{R}^{(v 1)}$ picks up $-F_{1}^{(v)}(x)$ besides $F_{4}^{(v 1)}(x)$. Thus we have $Y(x) C^{(v)-1}=F_{1}^{(v)}(x)+F_{3}^{(v 1)}(x)+F_{4}^{(v 1)}(x)-F_{1}^{(v)}(x)$ $=F_{2}^{(v 1)}(x)$. 
Lemma 2.5. $F_{2}^{(v 1)}(x)$ can be continued analytically into $\mathscr{R}^{(v 1)}$ across $I I_{\varepsilon}^{(v 1)}$ and satisfies

$$
F_{2}^{(v 1)}(x)_{\alpha \beta}=F_{2}^{(v 2)}(x)_{\alpha \beta}+\sum_{\gamma=1}^{m} F_{2}^{(v 2)}(x)_{\alpha \gamma} \lambda_{\gamma \beta}^{(v 1)} .
$$

Proof. We divide $F_{3}^{(v)}$ into two parts: $F_{3}^{(v)}=F_{5}^{(v)}+F_{6}^{(v)}$ where $F_{5}^{(v)}$ (resp. $F_{6}^{(v)}$ ) contains the $x_{j}$-integrations on $I I_{\varepsilon}^{(v 1)}$ (resp. $I I_{\varepsilon}^{(v 2)}$ ). The analytic continuation of $F_{5}^{(v)}(x)=F_{5}^{(v 1)}(x)$ into $\mathscr{R}^{(v 2)}$ picks up a linear combination of $F_{2}^{(v 2)}(x)$ besides $F_{5}^{(v 2)}$ :

$$
F_{5}^{(v 1)}(x)_{\alpha \beta}=F_{5}^{(v 2)}(x)_{\alpha \beta}+\sum_{\gamma=1}^{m} F_{2}^{(v 2)}(x)_{\alpha \gamma} \lambda_{\gamma \beta}^{(v 1)}
$$

The analytic continuation of $F_{6}^{(v)}(x)=F_{6}^{(v 1)}(x)$ (resp. $F_{4}^{(v 1)}(x)$ ) coincides with $F_{6}^{(v 2)}(x)\left(\right.$ resp. $\left.F_{4}^{(v 2)}(x)\right)$. Thus we have (2.26).

Similarly we can proceed to the case $\mathscr{R}^{(v 3)}, \mathscr{R}^{(v 4)}$ and so on. Thus we have proved (M1)-(M5). In particular we have shown that

$$
Y(x) C^{(v)-1} S_{1}^{(v)} \cdots S_{l-1}^{(v)}=F_{2}^{(v l)}(x) .
$$

If we expand $\left(x-x_{0}\right) /\left(x_{j}-x\right)$ in the integrand of $F_{2}^{(v l)}(x)$, we obtain (2.21). (M6) follows from the single-valuedness of $Y(x)$ near $x=\infty$ and the estimate $|Y(x)|=O\left(|x|^{1-\rho}\right)(0<\rho<1)$ for $|x| \rightarrow \infty$. In fact, we can rewrite (2.1) as

$$
\begin{aligned}
Y(x)_{\alpha \beta}=\delta_{\alpha \beta} & +2 \pi i\left(x-x_{0}\right) \sum_{\mu, \kappa=1}^{n} \sum_{\alpha^{\prime}, \beta^{\prime}=1}^{m} \int_{I^{(\mu)}} d y \int_{I^{(\kappa)}} d y^{\prime} \\
& \times\left(C^{(\mu)-1}\right)_{\alpha \alpha^{\prime}} \frac{1}{2 \pi} \frac{i}{x_{0}-y} R_{\alpha^{\prime} \beta^{\prime}}^{\left(\mu v^{\prime}\right.}\left(y, y^{\prime}\right) \frac{1}{2 \pi} \frac{i}{y^{\prime}-x} C_{\beta^{\prime} \beta}^{(\kappa)} .
\end{aligned}
$$

Then we have

$$
\begin{aligned}
Y(\infty)_{\alpha \beta}=\delta_{\alpha \beta} & +\sum_{\mu, \kappa=1}^{n} \sum_{\alpha^{\prime}, \beta^{\prime}=1}^{m} \int_{I^{(\mu)}} d y \int_{I^{(\kappa)}} d y^{\prime} \\
& \times\left(C^{(\mu)-1}\right)_{\alpha \alpha^{\prime}} \frac{1}{2 \pi} \frac{i}{x_{0}-y} R_{\alpha^{\prime} \beta^{\prime}}^{(\mu \kappa)}\left(y, y^{\prime}\right) C_{\beta^{\prime} \beta}^{(\kappa)} .
\end{aligned}
$$

\section{§3. Clifford Operators}

In this section we shall give a field theoretic expression for the matrix $Y(x)$ of the previous section. $Y(x)$ can be written in the form

$$
Y(x)_{\alpha \beta}=2 \pi i\left(x-x_{0}\right) \frac{\left\langle\psi_{\alpha}^{*}\left(x_{0}\right) \varphi_{1}^{(1)} \cdots \varphi_{m}^{(1)} \cdots \varphi_{1}^{(n)} \cdots \varphi_{m}^{(n)} \psi_{\beta}(x)\right\rangle}{\left\langle\varphi_{1}^{(1)} \cdots \varphi_{m}^{(1)} \cdots \varphi_{1}^{(n)} \cdots \varphi_{m}^{(n)}\right\rangle}
$$


where $\psi_{\alpha}^{*}\left(x_{0}\right)$ and $\psi_{\beta}(x)$ are free fermion fields and $\varphi_{\gamma}^{(\mu)}(\mu=1, \ldots, n ; \gamma=1, \ldots, m)$ is a Clifford operator. Here we call an operator which belongs to the Clifford group a Clifford operator. We refer the reader to [1] (Chapter I) as for the generalities on the Clifford group. We also give several operator identities for our free field operators and Clifford operators.

We introduce several species of free fermion field: $\psi_{\alpha}(x), \psi_{\alpha}^{*}(x)\left(x \in \mathscr{R}_{0}\right)$, $\phi_{\alpha}^{(\mu)}(x), \phi_{\alpha}^{*(\mu)}(x)\left(x \in I_{\varepsilon}^{(\mu)}\right), \phi_{\alpha}^{(\mu l)}(x), \phi_{\alpha}^{*(\mu l)}(x)\left(x \in I I_{\alpha}^{(\mu l)}\right)$. The expectation value is defined as follows.

$$
\left\langle\phi_{1}(x) \phi_{2}\left(x^{\prime}\right)\right\rangle=0,\left\langle\phi_{1}^{*}(x) \phi_{2}^{*}\left(x^{\prime}\right)\right\rangle=0
$$

and

$$
\left\langle\phi_{1}^{*}(x) \phi_{2}\left(x^{\prime}\right)\right\rangle=-\left\langle\phi_{2}\left(x^{\prime}\right) \phi_{1}^{*}(x)\right\rangle
$$

if $\phi_{j}=\psi_{\alpha}, \phi_{\alpha}^{(\mu)}$ or $\phi_{\alpha}^{(\mu l)}$ and if $\phi_{j}^{*}=\psi_{\alpha}^{*}, \phi_{\alpha}^{*(\mu)}$ or $\phi_{\alpha}^{*(\mu l)}$.

$$
\begin{aligned}
& \left\langle\psi_{\alpha}^{*}(x) \psi_{\beta}\left(x^{\prime}\right)\right\rangle=\frac{1}{2 \pi} \frac{i}{x-x^{\prime}} \delta_{\alpha \beta} \quad\left(x \neq x^{\prime}\right) . \\
& \left\langle\psi_{\alpha}^{*}(x) \phi_{\beta}^{(\mu)}\left(x^{\prime}\right)\right\rangle=\left(C^{(\mu)-1}\right)_{\alpha \beta} \frac{1}{2 \pi} \frac{i}{x-x^{\prime}} . \\
& \left\langle\phi_{\alpha}^{*(\mu)}(x) \psi_{\beta}\left(x^{\prime}\right)\right\rangle=C_{\alpha \beta}^{(\mu)} \frac{1}{2 \pi} \frac{i}{x-x^{\prime}} . \\
& \left\langle\psi_{\alpha}^{*}(x) \phi_{\beta}^{(\mu l)}\left(x^{\prime}\right)\right\rangle=\left\langle\phi_{\alpha}^{*(\mu l)}(x) \psi_{\beta}\left(x^{\prime}\right)\right\rangle=0 . \\
& \left\langle\phi_{\alpha}^{*(\mu)}(x) \phi_{\beta}^{(v)}\left(x^{\prime}\right)\right\rangle=\left(1-\delta_{\mu v}\right)\left(C^{(\mu)} C^{(v)-1}\right)_{\alpha \beta} \frac{1}{2 \pi} \frac{i}{x-x^{\prime}} . \\
& \left\langle\phi_{\alpha}^{*(\mu)}(x) \phi_{\beta}^{(v l)}\left(x^{\prime}\right)\right\rangle=\left\langle\phi_{\alpha}^{*(\mu l)}(x) \phi_{\beta}^{(v)}\left(x^{\prime}\right)\right\rangle=0 . \\
& \left\langle\phi_{\alpha}^{*(\mu l)}(x) \phi_{\beta}^{\left(v l^{\prime}\right)}\left(x^{\prime}\right)\right\rangle=\delta_{\mu \nu} \delta_{l l^{\prime}} \lambda_{\alpha \beta}^{(\mu l)} \delta\left(x-x^{\prime}\right) .
\end{aligned}
$$

We note that $C^{(\mu)}$ and $\lambda_{\alpha \beta}^{(\mu l)}$ have been introduced in the previous section.

We define a Clifford operator $\varphi_{\alpha}^{(\mu)}$ using the kernel $R_{\alpha}^{(\mu)}\left(x, x^{\prime}\right)$ of (2.14):

$$
\varphi_{\alpha}^{(\mu)}=\exp \rho_{\alpha}^{(\mu)}, \rho_{\alpha}^{(\mu)}=\iint d x d x^{\prime} R_{\alpha}^{(\mu)}\left(x, x^{\prime}\right) \psi_{\alpha}^{(\mu)}(x) \psi_{\alpha}^{*(\mu)}\left(x^{\prime}\right)
$$

where $\psi_{\alpha}^{(\mu)}(x)=\phi_{\alpha}^{(\mu)}(x)+\sum_{l=1}^{2 r_{\mu}} \phi_{\alpha}^{\left(\mu_{l}\right)}(x)$ and $\psi_{\alpha}^{*(\mu)}(x)=\phi_{\alpha}^{*(\mu)}(x)+\sum_{l=1}^{2 r_{\mu}} \phi_{\alpha}^{*(\mu l)}(x)$. In (3.10), if $x$ and $x^{\prime}$ belong to the same contour, we take $x^{\prime}$ to be on the right bank of the contour for $x$.

Theorem 3.1. The quadratic kernel for the product of $\varphi_{\alpha}^{(\mu)}(\mu=1, \ldots, n$; $\alpha=1, \ldots, m)$ is given by $R_{\alpha \beta}^{(\mu v)}\left(x, x^{\prime}\right)$ of $(2.2)$. Namely we have

$$
\frac{\varphi_{1}^{(1)} \cdots \varphi_{m}^{(n)}}{\left\langle\varphi_{1}^{(1)} \cdots \varphi_{m}^{(n)}\right\rangle}=: \exp \rho:
$$




$$
\rho=\sum_{\mu, v=1}^{n} \sum_{\alpha, \beta=1}^{m} \iint d x d x^{\prime} R_{\alpha \beta}^{(\mu \nu)}\left(x, x^{\prime}\right) \psi_{\alpha}^{(\mu)}(x) \psi_{\beta}^{*(v)}\left(x^{\prime}\right) .
$$

Proof. We set

$$
K_{\alpha \beta}^{(\mu v)}\left(x, x^{\prime}\right)=\left\langle\psi_{\alpha}^{*(\mu)}(x) \psi_{\beta}^{(v)}\left(x^{\prime}\right)\right\rangle
$$

Then if $\mu \neq v$,

$$
K_{\alpha \beta}^{(\mu v)}\left(x, x^{\prime}\right)=\left(C^{(\mu)} C^{(v)-1}\right)_{\alpha \beta} \frac{1}{2 \pi} \frac{i}{x-x^{\prime}}
$$

where $x \in I_{c}^{(\mu)}$ and $x^{\prime} \in I_{\varepsilon}^{(v)}$. We have also

$$
K_{\alpha \beta}^{(\mu \mu)}\left(x, x^{\prime}\right)=\lambda_{\alpha \beta}^{(\mu l)} \delta\left(x-x^{\prime}\right)
$$

where $x, x^{\prime} \in I I_{\varepsilon}^{(\mu l)}\left(l=1, \ldots, 2 r_{\mu}\right)$. The direct application of Wick's theorem leads to the following expression for the kernel in question:

$$
\begin{aligned}
& \sum_{j=0}^{\infty} \int d x_{1} \cdots \int d x_{2 j} \sum_{v_{1}, \ldots, v_{J-1}=1}^{n} \sum_{\alpha_{1}, \ldots, \alpha_{j-1}=1}^{m} \\
& \quad \times R_{\alpha}^{\left(v_{0}\right)}\left(x, x_{1}\right) K_{\alpha \alpha_{1}}^{\left(v_{0} v_{1}\right)}\left(x_{1}, x_{2}\right) \cdots R_{\alpha_{j-1}}^{\left(v_{j-1}\right)}\left(x_{2 j-2}, x_{2 j-1}\right) \\
& \quad \times K_{\alpha_{j}-1 \beta}^{\left(v_{j}-1 v_{j}\right)}\left(x_{2 j-1}, x_{2 j}\right) R_{\beta}^{\left(v_{j}\right)}\left(x_{2 j}, x^{\prime}\right), \quad\left(v_{0}=\mu, v_{j}=v\right) .
\end{aligned}
$$

If $v_{k-1}=v_{k}, x_{2 k-1}$ can be integrated out. If $v_{k-1} \neq v_{k}$, the contour for $x_{2 k-1}$ is $I_{\varepsilon}^{\left(v_{k-1}\right)}$. The singularities of the integrand with respect to $x_{2 k-1}$, which is located outside of $I_{\varepsilon}^{\left(v_{k-1}\right)}$, are the pole at $x_{2 k}$ and the branch point at $\infty$. Since $t_{0, \alpha_{j-1}}^{\left(v_{k}-1\right)}$ is small, the contour for $x_{2 k-1}$ can be deformed into a circle around $x_{2 k}$. Taking the residue at $x_{2 k}$, we obtain the desired expression (2.2) for $R_{\alpha \beta}^{(\mu v)}\left(x, x^{\prime}\right)$.

By a similar argument we have

Theorem 3.2. The $m \times m$ matrix $Y(x)$ can be written as (3.1).

We extend the domain of existence for $\psi_{\alpha}(x)$ and $\psi_{\alpha}^{*}(x)$, which were defined, thus far, only for $x \in \mathscr{R}_{0}$. For each $\mu$ we divide $\boldsymbol{C}$ into three pieces: $\boldsymbol{C}=\mathscr{R}_{\varepsilon}^{(\mu+)} \cup I_{\varepsilon}^{(\mu)} \cup \mathscr{R}_{\varepsilon}^{(\mu-)}$, where $\mathscr{R}_{\varepsilon}^{(\mu+)}$ (resp. $\mathscr{R}_{\varepsilon}^{(\mu-)}$ ) is the inside (resp. outside) of $I_{\varepsilon}^{(\mu)}$. We define the following expectation values:

$$
\begin{aligned}
&\left\langle\psi_{\alpha}^{*}(x) \psi_{\beta}\left(x^{\prime}\right)\right\rangle=-\left\langle\psi_{\beta}\left(x^{\prime}\right) \psi_{\alpha}^{*}(x)\right\rangle=\delta_{\alpha \beta} \frac{1}{2 \pi} \frac{i}{x-x^{\prime}} \\
& \text { if } \quad x, x^{\prime} \in C \text { and } x \neq x^{\prime}, \\
&\left\langle\psi_{\alpha}^{*}(x) \phi_{\beta}^{(\mu)}\left(x^{\prime}\right)\right\rangle=-\left\langle\phi_{\beta}^{(\mu)}\left(x^{\prime}\right) \psi_{\alpha}^{*}(x)\right\rangle=\left(C^{(\mu)-1}\right)_{\alpha \beta} \frac{1}{2 \pi} \frac{i}{x-x^{\prime}} \\
& \text { if } x \in \mathscr{R}_{\varepsilon}^{(\mu+)} \cup \mathscr{R}_{\varepsilon}^{(\mu-)} .
\end{aligned}
$$

If $x \in I_{c}^{(\mu)}\left\langle\psi_{\alpha}^{*}(x) \phi_{\beta}^{(\mu)}\left(x^{\prime}\right)\right\rangle$ (resp. $\left.\left\langle\phi_{\beta}^{(\mu)}\left(x^{\prime}\right) \psi_{\alpha}^{*}(x)\right\rangle\right)$ is defined to be the boundary 
value of $\left\langle\psi_{\alpha}^{*}(x) \phi_{\beta}^{(\mu)}\left(x^{\prime}\right)\right\rangle$ with $x \in \mathscr{R}_{\varepsilon}^{(\mu-)}$ (resp. $\left\langle\phi_{\beta}^{(\mu)}\left(x^{\prime}\right) \psi_{\alpha}^{*}(x)\right\rangle$ with $x \in \mathscr{R}_{\varepsilon}^{(\mu+)}$ ),

$$
\begin{aligned}
&\left\langle\phi_{\alpha}^{*(\mu)}(x) \psi_{\beta}\left(x^{\prime}\right)\right\rangle=-\left\langle\psi_{\beta}\left(x^{\prime}\right) \phi_{\alpha}^{*(\mu)}(x)\right\rangle=C_{\alpha \beta}^{(\mu)} \frac{1}{2 \pi} \frac{i}{x-x^{\prime}} \\
& \text { if } x \in \mathscr{R}_{\varepsilon}^{(\mu+)} \cup \mathscr{R}_{\varepsilon}^{(\mu-)} .
\end{aligned}
$$

If $x^{\prime} \in I_{\varepsilon}^{(\mu)},\left\langle\phi_{\alpha}^{*(\mu)}(x) \psi_{\beta}\left(x^{\prime}\right)\right\rangle$ (resp. $\left.\left\langle\psi_{\beta}\left(x^{\prime}\right) \phi_{\alpha}^{*(\mu)}(x)\right\rangle\right)$ is defined to be the boundary value of $\left\langle\phi_{\alpha}^{*(\mu)}(x) \psi_{\beta}\left(x^{\prime}\right)\right\rangle$ with $x^{\prime} \in \mathscr{R}_{\varepsilon}^{(\mu-)}$ (resp. $\left\langle\psi_{\beta}\left(x^{\prime}\right) \phi_{\alpha}^{*(\mu)}(x)\right\rangle$ with $x^{\prime} \in \mathscr{R}_{\varepsilon}^{(\mu+)}$ ).

Note the following identities:

$$
\begin{array}{ll}
{\left[\psi_{\alpha}^{*}(x), \phi_{\beta}^{(\mu)}\left(x^{\prime}\right)\right]_{+}=-\left(C^{(\mu)-1}\right)_{\alpha \beta} \delta\left(x-x^{\prime}\right)} & \text { if } x, x^{\prime} \in I_{r}^{(\mu)}, \\
{\left[\phi_{\alpha}^{*(\mu)}(x), \psi_{\beta}\left(x^{\prime}\right)\right]_{+}=C_{\alpha \beta}^{(\mu)} \delta\left(x-x^{\prime}\right)} & \text { if } x, x^{\prime} \in I_{c}^{(\mu)} .
\end{array}
$$

We also set

$$
\left\langle\phi_{1}(x) \phi_{2}\left(x^{\prime}\right)\right\rangle=0,\left\langle\phi_{1}^{*}(x) \phi_{2}^{*}\left(x^{\prime}\right)\right\rangle=0
$$

if $\phi_{j}=\psi_{\alpha}, \phi_{\alpha}^{(\mu)}$ or $\phi_{\alpha}^{(\mu l)}$ and if $\phi_{j}^{*}=\psi_{\alpha}^{*}, \phi_{\alpha}^{*(\mu)}$ or $\phi_{\alpha}^{*(\mu l)}$,

$$
\left\langle\phi_{1}^{*}(x) \phi_{2}\left(x^{\prime}\right)\right\rangle=0,\left\langle\phi_{2}(x) \phi_{1}^{*}(x)\right\rangle=0
$$

if $\phi_{1}^{*}=\psi_{\alpha}^{*}$ and $\phi_{2}=\phi_{\beta}^{(\mu l)}$ or if $\phi_{1}^{*}=\phi_{\alpha}^{*(\mu l)}$ and $\phi_{2}=\psi_{\beta}$.

We set

$$
e_{\alpha, k}^{(\mu)}(x)=\left(x-a_{\mu}\right)^{k} e_{\alpha}^{(\mu)}(x) \quad(k \in \mathbb{Z}),
$$

and define the following operators:

$$
\begin{aligned}
& \psi_{\alpha, l}^{(\mu)}=\frac{1}{2 \pi i} \int d x e_{\alpha, l}^{(\mu)}(x)^{-1} \psi_{\alpha}^{(\mu)}(x) . \\
& \psi_{\alpha,-k}^{*(\mu)}=\frac{1}{2 \pi i} \int d x e_{\alpha,-k}^{(\mu)}(x) \psi_{\alpha}^{*(\mu)}(x) . \\
& \varphi_{\alpha, l}^{(\mu)}=\psi_{\alpha, l}^{(\mu)} \varphi_{\alpha}^{(\mu)} . \\
& \varphi_{\alpha,-k}^{*(\mu)}=\psi_{\alpha,-k}^{*(\mu)} \varphi_{\alpha}^{(\mu)} . \\
& \varphi_{\alpha,-k l}^{(\mu)}=\psi_{\alpha,-k}^{*(\mu)} \psi_{\alpha, l}^{(\mu)} \varphi_{\alpha}^{(\mu)} .
\end{aligned}
$$

From (3.19) and (3.20) we have

\section{Proposition 3.3.}

$$
\begin{aligned}
& {\left[\varphi_{\alpha}^{(\mu)}, \sum_{\gamma=1}^{m} \psi_{\gamma}(x)\left(C^{(\mu)-1}\right)_{\gamma \beta}\right]} \\
& \quad=\left\{\begin{array}{lll}
\delta_{\alpha \beta} \sum_{k=0}^{\infty} \varphi_{\alpha, k+1}^{(\mu)} e_{\alpha, k}^{(\mu)}(x) & \text { if } & x \in I_{\varepsilon}^{(\mu)}, \\
0 & \text { if } & x \in \mathscr{R}_{+}^{(\mu)} \cup \mathscr{R}_{-}^{(\mu)} .
\end{array}\right.
\end{aligned}
$$


(3.30) $\quad\left[\sum_{\gamma=1}^{m} C_{\alpha \gamma}^{(\mu)} \psi_{\gamma}^{*}(x), \varphi_{\beta}^{(\mu)}\right]$

$$
=\left\{\begin{array}{lll}
\delta_{\alpha \beta} \sum_{k=0}^{\infty} \varphi_{\alpha,-k-1}^{*(\mu)} e_{\alpha,-k}^{(\mu)}(x)^{-1} & \text { if } & x \in I_{\varepsilon}^{(\mu)}, \\
0 & \text { if } & x \in \mathscr{R}_{+}^{(\mu)} \cup \mathscr{R}_{-}^{(\mu)} .
\end{array}\right.
$$

(3.31) $\left[\varphi_{\alpha,-k}^{*(\mu)}, \sum_{\gamma=1}^{m} \psi_{\gamma}(x)\left(C^{(\mu)-1}\right)_{\gamma \beta}\right]_{+}$

$$
=\left\{\begin{array}{lll}
\delta_{\alpha \beta}\left(\frac{1}{2 \pi i} \varphi_{\alpha}^{(\mu)} e_{\alpha,-k}^{(\mu)}(x)+\sum_{l=0}^{\infty} \varphi_{\alpha,-k l+1}^{(\mu)} e_{\alpha, l}^{(\mu)}(x)\right) & \text { if } & x \in I_{\varepsilon}^{(\mu)}, \\
0 & \text { if } & x \in \mathscr{R}_{+}^{(\mu)} \cup \mathscr{R}_{-}^{(\mu)} .
\end{array}\right.
$$

$$
\begin{aligned}
& {\left[\sum_{\gamma=1}^{m} C_{\alpha \gamma}^{(\mu)} \psi_{\gamma}^{*}(x), \varphi_{\beta, l}^{(\mu)}\right]_{+}} \\
& \quad=\left\{\begin{array}{lr}
\delta_{\alpha \beta}\left(-\frac{1}{2 \pi i} \varphi_{\alpha}^{(\mu)} e_{\alpha, l}^{(\mu)}(x)^{-1}+\sum_{k=0}^{\infty} \varphi_{\alpha,-k-1 l}^{(\mu)} e_{\alpha,-k}^{(\mu)}(x)^{-1}\right) & \text { if } x \in I_{\varepsilon}^{(\mu)}, \\
0 & \text { if } \quad x \in \mathscr{R}_{+}^{(\mu)} \cup \mathscr{R}_{-}^{(\mu)}
\end{array}\right.
\end{aligned}
$$

The derivatives of our field operators can be calculated by using the following formulas.

\section{Proposition 3.4.}

$$
\begin{aligned}
& \frac{\partial \psi_{\alpha, l}^{(\mu)}}{\partial a_{\mu}}=\sum_{j=0}^{r_{\mu}} t_{-j, \alpha}^{(\mu)} \psi_{\alpha, j+l+1}^{(\mu)}+k \psi_{\alpha, l+1}^{(\mu)}, \\
& \frac{\partial \psi_{\alpha, l}^{(\mu)}}{\partial t_{-j, \alpha}^{(\mu)}}=\frac{1}{j} \psi_{\alpha, j+l}^{(\mu)}, \\
& \frac{\partial \psi_{\alpha,-k}^{*(\mu)}}{\partial a_{\mu}}=-\sum_{j=0}^{r_{\mu}} t_{-j, \alpha}^{(\mu)} \psi_{\alpha,-j-k-1}^{*(\mu)}+k \psi_{\alpha,-k-1}^{*(\mu)}, \\
& \frac{\partial \psi_{\alpha,-k}^{*(\mu)}}{\partial t_{-j, \alpha}^{(\mu)}}=-\frac{1}{j} \psi_{\alpha,-j-k}^{* \alpha}, \\
& \frac{\partial \varphi_{\alpha}^{(\mu)}}{\partial a_{\mu}}=2 \pi i \sum_{j=0}^{r_{\mu}} \sum_{k=0}^{j} t_{-j, \alpha}^{(\mu)} \varphi_{\alpha, k-j-1 k+1}^{(\mu)}, \\
& \frac{\partial \varphi_{\alpha}^{(\mu)}}{\partial t_{-j, \alpha}^{(\mu)}}=2 \pi i \frac{1}{j} \sum_{k=0}^{j-1} \varphi_{\alpha, k-j k+1}^{(\mu)} .
\end{aligned}
$$

We denote by $\mathscr{T}_{\alpha}^{(\mu)}(\rho)$ the translation

$$
t_{-j, \alpha}^{(\mu)} \longmapsto t_{-j, \alpha}^{(\mu)}+\rho^{j}
$$

Proposition 3.5. Let us denote by $\equiv$ the congruence modulo $\rho^{r_{\mu}+1}$. Then we have

$$
\mathscr{T}_{\alpha}^{(\mu)}(\rho) \psi_{\alpha, l}^{(\mu)} \equiv \sum_{l^{\prime}=0}^{\infty} \rho^{l^{\prime}} \psi_{\alpha, l+l^{\prime}}^{(\mu)}
$$




$$
\begin{aligned}
& \mathscr{T}_{\alpha}^{(\mu)}(-\rho) \psi_{\alpha, l}^{(\mu)} \equiv \psi_{\alpha, l}^{(\mu)}-\rho \psi_{\alpha, l+1}^{*(\mu)}, \\
& \mathscr{T}_{\alpha}^{(\mu)}(\rho) \psi_{\alpha,-k}^{*(\mu)} \equiv \psi_{\alpha,-k}^{*(\mu)}-\rho \psi_{\alpha,-k-1}^{*(\mu)}, \\
& \mathscr{T}_{\alpha}^{(\mu)}(-\rho) \psi_{\alpha,-k}^{*(\mu)} \equiv \sum_{k^{\prime}=0}^{\infty} \rho^{k^{\prime}} \psi_{\alpha,-k-k^{\prime}}^{*(\mu)}, \\
& \mathscr{T}_{\alpha}^{(\mu)}(\rho) \varphi_{\alpha}^{(\mu)} \equiv \varphi_{\alpha}^{(\mu)}+2 \pi i \sum_{l=1}^{\infty} \rho^{l} \varphi_{\alpha,-1 l}^{(\mu)},
\end{aligned}
$$

and

$$
\mathscr{T}_{\alpha}^{(\mu)}(-\rho) \varphi_{\alpha}^{(\mu)} \equiv \varphi_{\alpha}^{(\mu)}-2 \pi i \sum_{k=1}^{\infty} \rho^{k} \varphi_{\alpha,-k 1}^{(\mu)}
$$

Let $\boldsymbol{x}=\left(x_{1}, x_{2}, x_{3}, \ldots\right)$ be a ordered set of infinite variables. We define a polynomial $P_{j}(x)$ of $x_{1}, \ldots, x_{j}$ by

$$
\exp \left(\sum_{j=1}^{\infty} \rho^{j} \cdot x_{j}\right)=\sum_{j=1}^{\infty} \rho^{j} P_{j}(x)
$$

Proposition 3.6. We set $\partial_{\alpha}^{(\mu)}=\left(\partial / \partial t_{-1, \alpha}^{(\mu)}, \partial / \partial t_{-2, \alpha}^{(\mu)}, \ldots, \partial / \partial t_{-r_{\mu, \alpha}}^{(\mu)}, 0, \ldots\right)$. Then we have

$$
\begin{array}{ll}
\varphi_{\alpha, l+1}^{(\mu)}=P_{l}\left(\partial_{\alpha}^{(\mu)}\right) \varphi_{\alpha, 1}^{(\mu)} & 1 \leqq l \leqq r_{\mu}, \\
\varphi_{\alpha,-k-1}^{*(\mu)}=P_{k}\left(-\partial_{\alpha}^{(\mu)}\right) \varphi_{\alpha,-1}^{*(\mu)} & 1 \leqq k \leqq r_{\mu},
\end{array}
$$

and

$$
\begin{aligned}
\varphi_{\alpha,-k l}^{(\mu)} & =\sum_{j=0}^{k-1} P_{j}\left(-\partial_{\alpha}^{(\mu)}\right) P_{k+l-1-j}\left(\partial_{\alpha}^{(\mu)}\right) \varphi_{\alpha}^{(\mu)} \\
& =-\sum_{j=0}^{l-1} P_{k+l-1-j}\left(-\partial_{\alpha}^{(\mu)}\right) P_{j}\left(\partial_{\alpha}^{(\mu)}\right) \varphi_{\alpha}^{(\mu)} \quad 1 \leqq l, k \leqq r_{\mu} .
\end{aligned}
$$

We omit the proof of Propositions 3.3-3.6 since it is a straightforward calculation. We only note that we use the following property of a free fermion.

Lemma 3.7. Let $f(x)$ and $f^{*}(x)$ be arbitrary functions on $I_{\varepsilon}^{(\mu)} \cup I I_{\varepsilon}^{(\mu, 1)}$ $\cup \cdots \cup I I_{\varepsilon}^{\left(\mu, 2 r_{\mu}\right)}$. Then we have

$$
\left(\int d x f(x) \psi_{\alpha}^{(\mu)}(x)+\int d x f^{*}(x) \psi_{\alpha}^{*(\mu)}(x)\right)^{2}=0
$$

\section{§4. Wave Function}

A function of $x$ is called a wave function if its $x$-dependence comes from a free field in the expectation value. In (3.1) we gave an example of a wave func- 
tion which solves the Riemann's monodromy problem. In this section we exploit the operator identities to study wave functions in detail. As a byproduct we obtain operator expressions for the characteristic matrices and the Schlesinger transforms [10].

We consider the following wave function.

$$
\begin{aligned}
& Y_{k}^{(\mu)}(x)_{\alpha \beta}=2 \pi i\left(. x-a_{\mu}\right)^{k} \\
& \quad \times \frac{\left\langle\varphi_{1}^{(1)} \cdots \varphi_{1}^{(\mu)} \cdots \varphi_{\alpha,-k}^{*(\mu)} \cdots \varphi_{m}^{(\mu)} \cdots \varphi_{m}^{(n)} \psi_{\beta}(x)\right\rangle}{\left\langle\varphi_{1}^{(1)} \cdots \varphi_{m}^{(n)}\right\rangle} \quad(k \geqq 1) .
\end{aligned}
$$

By the same argument as in Sections 2 and 3 we can show that $Y_{k}^{(\mu)}(x)=$ $\left(Y_{k}^{(\mu)}(x)_{\alpha \beta}\right)_{\alpha \beta=1, \ldots, m}$ satisfies (M1), (M3), (M4) and (M5). Moreover it is so normalized that

$$
\begin{array}{ll}
Y_{k}^{(\mu)}(x)=O\left(|x|^{k-1}\right) & |x| \longrightarrow \infty, \\
\left(Y_{k}^{(\mu)}(x) C^{(\mu)-1}\right)_{\alpha \beta} e_{\beta}^{(\mu)}(x)^{-1} \sim \delta_{\alpha \beta}+O\left(\left|x-a_{\mu}\right|^{k}\right) & |x-a| \longrightarrow 0 .
\end{array}
$$

In fact we have

$$
\begin{aligned}
Y_{k}^{(\mu)}(x)_{\alpha \beta}= & e_{\alpha}^{(\mu)}(x) C_{\alpha \beta}^{(\mu)}-\left(x-a_{\mu}\right)^{k} \sum_{v_{1}, v_{2}=1}^{n} \sum_{\alpha_{1}, \alpha_{2}=1}^{m} \int d x_{1} \int d x_{2} \\
& \times e_{\alpha,-k}^{(\mu)}\left(x_{1}\right) K_{\alpha \alpha_{1}}^{\left(\mu v_{1}\right)}\left(x_{1}, x_{2}\right) R_{\alpha_{1} \alpha_{2}}^{\left(v_{1} v_{2}\right)}\left(x_{2}, x\right) C_{\alpha_{2} \beta}^{\left(v_{2}\right)} .
\end{aligned}
$$

Theorem 4.1. The monodromy properties (M1), (M3), (M4), (M5) and the normalization (4.2), (4.3) uniquely determines the matrix $Y_{k}^{(\mu)}(x)$, if it ever exists. If the parameters $t_{-j, \alpha}^{(\mu)}$ and $\lambda_{\alpha \beta}^{(\mu l)}\left(\mu=1, \ldots, n ; j=0,1, \ldots, r_{\mu} ; \alpha, \beta=1, \ldots\right.$, $\left.m ; l=1, \ldots, 2 r_{\mu}\right)$ are sufficiently small, such $Y_{k}^{(\mu)}(x)$ exists and it can be expressed as a wave function (4.1).

Proof. It is sufficient to prove the uniqueness. If $k=1$, the proof is similar to that of Proposition 2.1. $\left(Y_{1}^{(\mu)}(x) C^{(\mu)-1}\right)_{\alpha \beta} e_{\beta}^{(\mu)}(x)^{-1}$ has an asymptotic expansion of the form $\hat{Y}^{(\mu)}(x)_{\alpha \beta}=\delta_{\alpha \beta}+O\left(\left|x-a_{\mu}\right|\right)$ at $x=a_{\mu}$. We define a polynomial $R_{k-1}^{(\mu)}(x)_{\alpha \beta}$ of degree $k-1$ by

$$
\left(\hat{Y}^{(\mu)}(x)^{-1}\right)_{\alpha \beta} \equiv R_{k-1}^{(\mu)}(x)_{\alpha \beta} \quad\left(\bmod \left|x-a_{\mu}\right|^{k}\right) .
$$

Now from (4.2) and (4.3) we can easily show that

$$
Y_{k}^{(\mu)}(x)=R_{k-1}^{(\mu)}(x) Y_{1}^{(\mu)}(x) .
$$

Thus $Y_{k}^{(\mu)}(X)$ is also unique.

Theorem 4.2. At $x=a_{v}, Y_{k}^{(\mu)}(x)$ has the following asymptotic expansion:

$$
\left(Y_{k}^{(\mu)}(x) C^{(v)-1}\right)_{\alpha \beta} e_{\beta}^{(v)}(x)^{-1} \sim \sum_{l \in Z} G_{\alpha \beta}^{(\mu v)(k l)}\left(x-a_{\mu}\right)^{k}\left(x-a_{v}\right)^{l-1} .
$$


If $k, l \geqq 1$, we have

$$
\begin{aligned}
& G_{\alpha \beta}^{(\mu v)(k l)} \\
& = \begin{cases}-2 \pi i \frac{\left\langle\varphi_{1}^{(1)} \cdots \varphi_{\beta, l}^{(\nu)} \cdots \varphi_{\alpha,-k}^{*(\mu)} \cdots \varphi_{m}^{(n)}\right\rangle}{\left\langle\varphi_{1}^{(1)} \cdots \varphi_{m}^{(n)}\right\rangle} & \text { if } \mu>v \text { or } \mu=v, \alpha>\beta, \\
2 \pi i \frac{\left\langle\varphi_{1}^{(1)} \cdots \varphi_{\alpha,-h l}^{(\mu)} \cdots \varphi_{m}^{(n)}\right\rangle}{\left\langle\varphi_{1}^{(1)} \cdots \varphi_{m}^{(n)}\right\rangle} & \text { if } \mu=v, \alpha=\beta, \\
2 \pi i \frac{\left\langle\varphi_{1}^{(1)} \cdots \varphi_{\alpha,-k}^{*(\mu)} \cdots \varphi_{\beta, l}^{(\nu)} \cdots \varphi_{m}^{(n)}\right\rangle}{\left\langle\varphi_{1}^{(1)} \cdots \varphi_{m}^{(n)}\right\rangle} & \text { if } \mu<v \text { or } \mu=v, \alpha<\beta,\end{cases} \\
& =\frac{1}{2 \pi i} \int d x_{1} \int d x_{2} e_{\alpha,-k}^{(\mu)}\left(x_{1}\right) K_{\alpha \beta}^{(\mu \nu)}\left(x_{1}, x_{2}\right) e_{\beta, l}^{(v)}\left(x_{2}\right)^{-1} \\
& +\frac{1}{2 \pi i} \sum_{v_{1}, v_{2}=1}^{n} \sum_{\alpha_{1}, \alpha_{2}=1}^{m} \int d x_{1} \int d x_{2} \int d x_{3} \int d x_{4} \\
& \times e_{\alpha,-k}^{(\mu)}\left(x_{1}\right) K_{\alpha, \alpha_{1}}^{\left(\mu v_{1}\right)}\left(x_{1}, x_{2}\right) R_{\alpha_{1} \alpha_{2}}^{\left(v_{1} v_{2}\right)}\left(x_{2}, x_{3}\right) K_{\alpha_{2} \beta}^{\left(v_{2} v\right)}\left(x_{3}, x_{4}\right) e_{\beta, l}^{(v)}\left(x_{4}\right)^{-1} .
\end{aligned}
$$

If $l \leqq 0, G_{\alpha \beta}^{(\mu \nu)(k l)}=\delta_{k, 1-l} \delta_{\alpha \beta}$.

Proof. One can prove Theorem 4.2 by following the argument in Section 2. If we substitute $Y(x)$ by $Y_{k}^{(\mu)}(x),(2.21)$ corresponds to the second expression of $G_{\alpha \beta}^{(\mu \nu)(k l)}$ in (4.8), then the first expression follows from Wick's theorem. A direct way to show (4.8) is to exploit (3.29) and (3.31). Assume that $\mu<\nu$ or $\mu=v, \alpha<\beta$. If $x \in I_{\varepsilon}^{(\mu)}$, the boundary value of $\left(Y_{k}^{(\mu)}(x) C^{(v)-1}\right)_{\alpha \beta}$ from $\mathscr{R}_{0}$ is given by

$$
2 \pi i\left(x-a_{\mu}\right)^{h} \frac{\left\langle\varphi_{1}^{(1)} \cdots \varphi_{\alpha,-k}^{*(\mu)} \cdots \varphi_{\beta}^{(v)}\left(\sum_{\gamma=1}^{m} \psi_{\gamma}(x)\left(C^{(v)-1}\right)_{\gamma \beta}\right) \cdots \varphi_{m}^{(n)}\right\rangle}{\left\langle\varphi_{1}^{(1)} \cdots \varphi_{m}^{(n)}\right\rangle},
$$

while the boundary value of $F_{1}^{(v)}(x)$ from $\mathscr{R}^{(v 1)}$ is given by

$$
2 \pi i\left(x-a_{\mu}\right)^{k} \frac{\left\langle\varphi_{1}^{(1)} \cdots \varphi_{\alpha,-k}^{*(\mu)} \cdots\left(\sum_{\gamma=1}^{m} \psi_{\gamma}(x)\left(C^{(v)-1}\right)_{\gamma \beta}\right) \varphi_{\beta}^{(v)} \cdots \varphi_{m}^{(n)}\right\rangle}{\left\langle\varphi_{1}^{(1)} \cdots \varphi_{m}^{(n)}\right\rangle} .
$$

Thus $F_{2}^{(v)}(x)$ is given by

$$
2 \pi i\left(x-a_{\mu}\right)^{k} \frac{\left\langle\varphi_{1}^{(1)} \cdots \varphi_{\alpha,-k}^{*(\mu)} \cdots\left[\varphi_{\beta}^{(v)},\left(\sum_{\gamma=1}^{m} \psi_{\gamma}(x)\left(C^{(v)-1}\right)_{\gamma \beta}\right)\right] \cdots \varphi_{m}^{(n)}\right\rangle}{\left\langle\varphi_{1}^{(1)} \cdots \varphi_{m}^{(n)}\right\rangle} .
$$

Hence using (3.29) we obtain (4.8).

The matrices $G^{(\mu v)(k l)}$ were introduced in [10], where they are called the characteristic matrices. The following differential equations for the characteristic matrices are known [10]. We can derive them using the infinite series ex- 
pressions (4.8) for $G_{\alpha \beta}^{(\mu \nu)(k l)}$. Here we shall give another proof by exploiting their operator expressions.

\section{Theorem 4.3.}

$$
\begin{aligned}
\frac{\partial G_{\alpha \beta}^{(\mu \nu)(k l)}}{\partial a_{\lambda}}= & -\sum_{\gamma=1}^{m} \sum_{j=0}^{r_{\lambda}} \sum_{s \in Z} t_{-j \gamma}^{(\mu)} G_{\alpha \gamma}^{(\mu \lambda)(k s+1)} G_{\gamma \beta}^{(\lambda v)(-s+j+1 l)} \\
& +k G_{\alpha \beta}^{(\mu v)(k+1 l)} \delta_{\mu \lambda}+l G_{\alpha \beta}^{(\mu v)(k l+1)} \delta_{v \lambda} . \\
\frac{\partial G_{\alpha \beta}^{(\mu \nu)(k l)}}{\partial t_{-j \alpha}^{(\lambda)}}= & -\frac{1}{j} \sum_{s \in Z} G_{\alpha \gamma}^{(\mu \lambda)(k s+1)} G_{\lambda \beta}^{(\lambda v)(-s+j l)} .
\end{aligned}
$$

Proof. We restrict ourselves to the case $\mu=\lambda<v$ in (4.12):

$$
\begin{aligned}
& \frac{\partial G_{\alpha \beta}^{(\mu \nu)(k l)}}{\partial a_{\mu}}= 2 \pi i \frac{\partial}{\partial a_{\mu}}\left(\frac{\left\langle\varphi_{1}^{(1)} \cdots \psi_{\alpha,-k}^{*(\mu)} \varphi_{\alpha}^{(\mu)} \cdots \varphi_{\beta}^{(v)} \cdots \varphi_{m}^{(n)}\right\rangle}{\left\langle\varphi_{1}^{(1)} \cdots \varphi_{m}^{(n)}\right\rangle}\right) \\
&= 2 \pi i \frac{\left\langle\varphi_{1}^{(1)} \cdots \frac{\partial \psi_{\alpha, k}^{*(\mu)}}{\partial a_{\mu}} \varphi_{\alpha}^{(\mu)} \cdots \varphi_{\beta, l}^{(\nu)} \cdots \varphi_{m}^{(n)}\right\rangle}{\left\langle\varphi_{1}^{(1)} \cdots \varphi_{m}^{(n)}\right\rangle} \\
&+2 \pi i \sum_{\gamma=1}^{m}\left(\frac{\left\langle\varphi_{1}^{(1)} \cdots \psi_{\alpha,-k}^{*(\mu)} \frac{\partial \varphi_{\gamma}^{(\mu)}}{\partial a_{\mu}} \cdots \varphi_{\beta, l}^{(v)} \cdots \varphi_{m}^{(n)}\right\rangle}{\left\langle\varphi_{1}^{(1)} \cdots \varphi_{m}^{(n)}\right\rangle}\right. \\
&\left.-\frac{\left\langle\varphi_{1}^{(1)} \cdots \psi_{\alpha,-k}^{*(\mu)} \varphi_{\alpha}^{(\mu)} \cdots \varphi_{\beta, l}^{(\nu)} \cdots \varphi_{m}^{(n)}\right\rangle}{\left\langle\varphi_{1}^{(1)} \cdots \varphi_{m}^{(n)}\right\rangle} \cdot \frac{\left\langle\varphi_{1}^{(1)} \cdots \psi_{\alpha,-k}^{*(\mu)} \varphi_{\alpha}^{(\mu)} \cdots \frac{\partial \varphi_{\gamma}^{(\mu)}}{\partial a_{\mu}} \cdots \varphi_{m}^{(n)}\right\rangle}{\left\langle\varphi_{1}^{(1)} \cdots \varphi_{m}^{(n)}\right\rangle}\right)
\end{aligned}
$$

Here we use Proposition 3.4 and the following formula (see [1] Chapter IV, Appendix):

$$
\begin{gathered}
\frac{\left\langle\varphi_{1}^{(1)} \cdots \psi_{\alpha,-k}^{*(\mu)} \varphi_{\alpha}^{(\mu)} \cdots \psi_{\gamma, k-j-1}^{*(\mu)} \psi_{\gamma, k+1}^{(\mu)} \varphi_{\gamma}^{(\mu)} \cdots \psi_{\beta, l}^{(v)} \varphi_{\beta}^{(v)} \cdots \varphi_{m}^{(n)}\right\rangle}{\left\langle\varphi_{1}^{(1)} \cdots \varphi_{m}^{(n)}\right\rangle} \\
=\frac{\left\langle\varphi_{1}^{(1)} \cdots \psi_{\alpha,-k}^{*(\mu)} \varphi_{\alpha}^{(\mu)} \cdots \psi_{\beta, l}^{(v)} \varphi_{\beta}^{(v)} \cdots \varphi_{m}^{(n)}\right\rangle}{\left\langle\varphi_{1}^{(1)} \cdots \varphi_{m}^{(n)}\right\rangle} \cdot \frac{\left\langle\varphi_{1}^{(1)} \cdots \psi_{\gamma, k-j+1}^{*(\mu)} \psi_{\gamma, k+1}^{(\mu)} \varphi_{\gamma}^{(\mu)} \cdots \varphi_{m}^{(n)}\right\rangle}{\left\langle\varphi_{1}^{(1)} \cdots \varphi_{m}^{(n)}\right\rangle} \\
-\frac{\left\langle\varphi_{1}^{(1)} \cdots \psi_{\alpha,-k}^{*(\mu)} \varphi_{\alpha}^{(\mu)} \cdots \psi_{\gamma, k+1}^{(\mu)} \varphi_{\gamma}^{(\mu)} \cdots \varphi_{m}^{(n)}\right\rangle}{\left\langle\varphi_{1}^{(1)} \cdots \varphi_{m}^{(n)}\right\rangle} \\
\times \frac{\left\langle\varphi_{1}^{(1)} \cdots \psi_{\gamma, k-j-1}^{*(\mu)} \varphi_{\gamma}^{(\mu)} \cdots \psi_{\beta, l}^{(v)} \varphi_{\beta}^{(v)} \cdots \varphi_{m}^{(n)}\right\rangle}{\left\langle\varphi_{1}^{(1)} \cdots \varphi_{m}^{(n)}\right\rangle} .
\end{gathered}
$$

Then noting that $G_{\alpha \beta}^{(\mu \mu)(k,-k+1)}=\delta_{\alpha \beta}(k \geqq 1)$ we obtain (4.12).

Let us consider the Schlesinger transformation [10] of wave functions. Let $l_{\alpha}^{(\mu)}(\mu=1, \ldots, n ; \alpha=1, \ldots, m)$ be integers satisfying $\sum_{\mu=1}^{n} \sum_{\alpha=1}^{m} l_{\alpha}^{(\mu)}=0$. We set as follows: 
(4.14)

$$
\varphi_{\alpha}^{(\mu, s)}=\left\{\begin{array}{l}
\psi_{\alpha,-1}^{*(\mu)} \cdots \psi_{\alpha, s}^{*(\mu)} \varphi_{\alpha}^{(\mu)} \\
\psi_{\alpha, 1}^{(\mu)} \cdots \psi_{\alpha, s}^{(\mu)} \varphi_{\alpha}^{(\mu)}
\end{array}\right.
$$

if $s \leqq 0$,

if $0 \leqq s$.

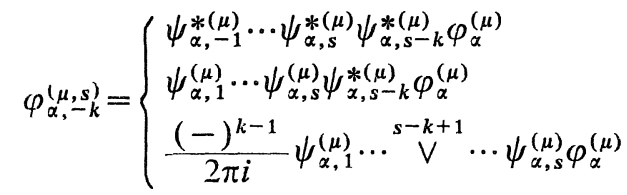

if $s \leqq 0$,

if $0 \leqq s<k$,

if $k \leqq s,(k \geqq 1)$.

(4.16)

$\begin{aligned} & \varphi_{\alpha, l}^{(\mu, s)}= \begin{cases}\frac{(-)^{l-1}}{2 \pi i} \psi_{\alpha,-1}^{*(\mu)} \cdots \stackrel{1-s-l}{\vee} \cdots \psi_{\alpha, s}^{*(\mu)} \varphi_{\alpha}^{(\mu)} & \text { if } s \leqq-l, \\ \psi_{\alpha,-1}^{*(\mu)} \cdots \psi_{\alpha, s}^{*(\mu)} \psi_{\alpha, s+l}^{(\mu)} \varphi_{\alpha}^{(\mu)} & \text { if }-l<s \leqq 0, \\ \psi_{\alpha, 1}^{(\mu)} \cdots \psi_{\alpha, s}^{(\mu)} \psi_{\alpha, s+l}^{(\mu)} \varphi_{\alpha}^{(\mu)} & \text { if } 0 \leqq s,(l \geqq 1) .\end{cases} \\ & \varphi_{\alpha,-k l}^{(\mu, s)}= \begin{cases}\frac{(-)^{l}}{2 \pi i} \psi_{\alpha,-1}^{*(\mu)} \cdots \stackrel{1-s-l}{V} \cdots \psi_{\alpha, s}^{*(\mu)} \psi_{\alpha, s-k}^{*(\mu)} \varphi_{\alpha}^{(\mu)} & \text { if } s \leqq-l, \\ \psi_{\alpha,-1}^{*(\mu)} \cdots \psi_{\alpha, s}^{*(\mu)} \psi_{\alpha, s-k}^{*(\mu)} \psi_{\alpha, s+l}^{(\mu)} \varphi_{\alpha}^{(\mu)} & \text { if }-l<s \leqq 0, \\ \psi_{\alpha, 1}^{(\mu)} \cdots \psi_{\alpha, s}^{(\mu)} \psi_{\alpha, s-k}^{*(\mu)} \psi_{\alpha, s+l}^{(\mu)} \varphi_{\alpha}^{(\mu)} & \text { if } 0 \leqq s<k, \\ \frac{(-)^{k-1}}{2 \pi i} \psi_{\alpha, 1}^{(\mu)} \cdots{ }^{-k+k+1} \cdots \psi_{\alpha, s}^{(\mu)} \psi_{\alpha, s+l}^{(\mu)} \varphi_{\alpha}^{(\mu)} & \text { if } k \leqq s,(l, k \geqq 1) .\end{cases} \end{aligned}$

Theorem 4.4. The following wave function $Y^{\prime}(x)=\left(Y^{\prime}(x)_{\alpha \beta}\right)_{\alpha, \beta=1, \ldots, m}$ satisfies (M1)-(M6) with $t_{0 \alpha}^{(\mu)}$ replaced by $l_{0, \alpha}^{(\mu)}+t_{\alpha}^{(\mu)}$.

Proof. (M2) is obvious. We set $N=\sum_{l_{\alpha}}^{(\mu)}>0 l_{\alpha}^{(\mu)}$. We define an $N \times N$ matrix $W$ and an $(N+1) \times(N+1)$ matrix $W(x ; \alpha \beta)$ by

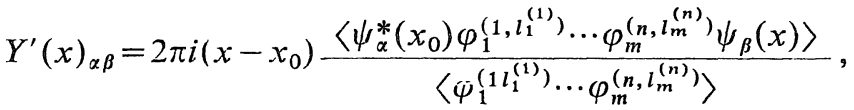

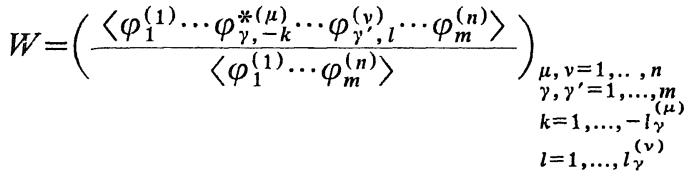

and

$$
=\left(\begin{array}{cc}
\frac{\left\langle\psi_{\alpha}^{*}\left(x_{0}\right) \varphi_{1}^{(1)} \cdots \varphi_{m}^{(n)} \psi_{\beta}(x)\right\rangle}{\left\langle\varphi_{1}^{(1)} \cdots \varphi_{m}^{(n)}\right\rangle} & \left(\frac{\left\langle\psi_{\alpha}\left(x_{0}\right) \varphi_{1}^{(1)} \cdots \varphi_{\gamma, l}^{(\mu)} \cdots \varphi_{m}^{(n)}\right\rangle}{\left\langle\varphi_{1}^{(1)} \cdots \varphi_{m}^{(n)}\right\rangle}\right)_{\substack{\mu=1, \ldots, n \\
\gamma=1, \ldots, m \\
l=1, \ldots, l_{\gamma}^{(\mu)}}} \\
\left(\frac{\left\langle\varphi_{1}^{(1)} \cdots \varphi_{\gamma,-k}^{*(\mu)} \cdots \varphi_{m}^{(n)} \psi_{\beta}(x)\right\rangle}{\left\langle\varphi_{1}^{(1)} \cdots \varphi_{m}^{(n)}\right\rangle}\right)_{\substack{\mu=1, \ldots, n \\
\gamma=1, \ldots, m \\
k=1, \ldots,-l, l}} & W
\end{array}\right) .
$$

Then we have

$$
Y^{\prime}(x)_{\alpha \beta}=2 \pi i\left(x-x_{0}\right) \frac{\operatorname{det} W(x, \alpha \beta)}{\operatorname{det} W} .
$$


Hence $Y^{\prime}(x)$ is a linear combination of matrices satisfying (M1), (M3), (M4), (M5) and (M6) with $t_{0 \alpha}^{(\mu)}$ replaced by $t_{0 \alpha}^{(\mu)}$ plus a certain integer $l_{\alpha}^{\prime(\mu)}$. Using (3.29) we have

$$
\begin{aligned}
& {\left[\varphi_{\alpha}^{(\mu, s)}, \sum_{\gamma=1}^{m} \psi_{\gamma}(x)\left(C^{(\mu)-1}\right)_{\gamma \beta}\right]_{ \pm}} \\
& =\left\{\begin{array}{ccc}
\delta_{\alpha \beta} \sum_{l=0}^{\infty} \varphi_{\alpha, l+1}^{(\mu, s)} e_{\alpha, s+l}^{(\mu)}(x) & \text { if } & x \in I_{\varepsilon}^{(\mu)}, \\
0 & \text { if } & x \in \mathscr{R}_{+}^{(\mu)} \cup \mathscr{R}_{-}^{(\mu)} .
\end{array}\right.
\end{aligned}
$$

Here $[,]_{ \pm}$denote $[$,$] or [,]_{+}$according as $s$ is even or odd. This expansion shows that $l_{\alpha}^{(\mu)}=l_{\alpha}^{(\mu)}$ for our $Y^{\prime}(x)$.

Theorem 4.5. The following wave function satisfies (M1), (M3), (M4), (M5), (4.2) and (4.3) with $t_{0, \alpha}^{(\mu)}$ replaced by $t_{0, \alpha}^{(\mu)}+l_{\alpha}^{(\mu)}$ :

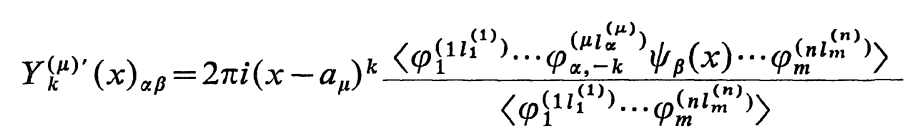

Proof. It is sufficient to check (4.3) with $t_{0, \alpha}^{(\mu)}$ replaced by $t_{0, \alpha}^{(\mu)}+l_{\alpha}^{(\mu)}$. From (3.29) we have

$$
\begin{aligned}
& {\left[\varphi_{\alpha,-k}^{(\mu s)}, \sum_{\gamma=1}^{m} \psi_{\gamma}(x)\left(C^{(\mu)-1)}\right)_{\gamma \beta}\right]_{ \pm}} \\
& \quad=\delta_{\alpha \beta}\left(\frac{1}{2 \pi i} \varphi_{\alpha}^{(\mu s)} e_{\alpha, s-k}^{(\mu)}(x)+\sum_{j=s}^{\infty} \varphi_{\alpha,-k, j+1-s}^{(\mu s)} e_{\alpha, j}^{(\mu)}(x)\right),
\end{aligned}
$$

which shows (4.3).

Theorem 4.6. At $x=a_{v} Y_{k}^{(\mu) \prime}(x)$ has the following asymptotic expansion:

$$
\left(Y_{k}^{(\mu)^{\prime}}(x) C^{(v)-1}\right)_{\alpha \beta} e_{\beta, s}^{(v)}(x)^{-1} \sim \sum_{l \in Z} G_{\alpha \beta}^{(\mu v)(k l)^{\prime}}\left(x-a_{\mu}\right)^{k}\left(x-a_{v}\right)^{l-1} .
$$

If $k, l \geqq 1$ we have

$$
\begin{aligned}
& G_{\alpha \beta}^{(\mu \nu)(k l)^{\prime}}
\end{aligned}
$$

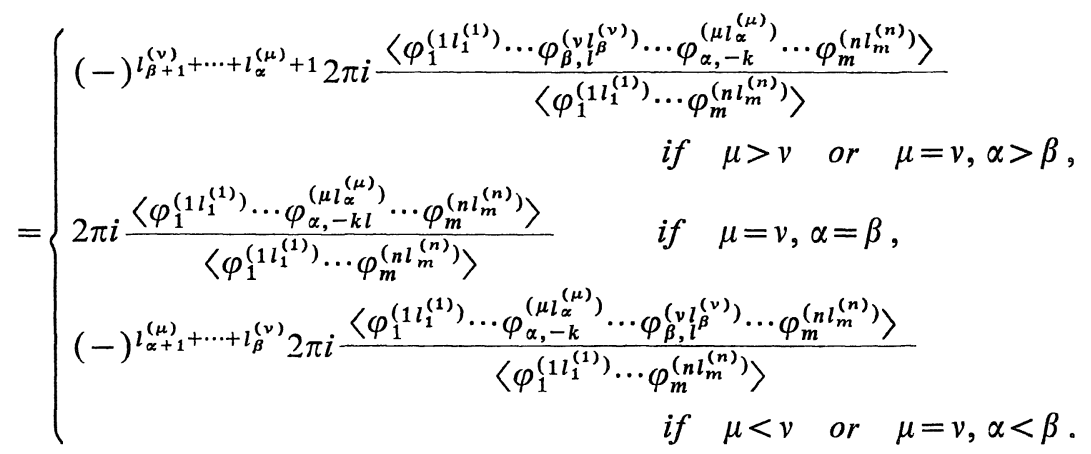


Proof. This is a direct consequence of (4.21) and (4.24).

Remark. (4.26) is equivalent to (17) in [20] II.

\section{\$5. Correlation Function}

In this section we shall prove that the correlation function $\left\langle\varphi_{1}^{(1)} \cdots \varphi_{m}^{(n)}\right\rangle$ of our Clifford operators $\varphi_{\alpha}^{(\mu)}(\mu=1, \ldots, n ; \alpha=1, \ldots, m)$ coincides with the $\tau$ function defined in [9]:

$$
\tau=\left\langle\varphi_{1}^{(1)} \cdots \varphi_{m}^{(n)}\right\rangle .
$$

The application of Wick's theorem leads us to the following Fredholm determinant for the correlation function.

$$
\begin{aligned}
& \left\langle\varphi_{1}^{(1)} \cdots \varphi_{m}^{(n)}\right\rangle=\sum_{l=0}^{\infty} \frac{(-)^{l}}{l !} \sum_{v_{1}, \ldots, v_{l}=1}^{n} \sum_{\alpha_{1}, \ldots, \alpha_{l}=1}^{m} \int d x_{1} \ldots \int d x_{l} \\
& \quad \times \operatorname{det}\left(\frac{1}{2 \pi i} \frac{1}{x_{j}-x_{k}} \frac{e_{\alpha_{j}}^{\left(v_{j}\right)}\left(x_{k}\right)}{e_{\alpha_{k}}^{\left(v_{k}\right)}\left(x_{k}\right)} K_{\alpha_{j} \alpha_{k}}^{\left(v_{j} v_{k}\right)}\right)_{j, k=1, \ldots, l}
\end{aligned}
$$

Unfortunately because of the infinite length of contours for integration (5.2) does not give a finite quantity. The following Neumann series defines instead the logarithmic derivative of the correlation function.

$$
\begin{aligned}
& d \log \left\langle\varphi_{1}^{\left.(1) \cdots \varphi_{m}^{(n)}\right\rangle}\right. \\
& =-\sum_{l=1}^{\infty} \sum_{v_{1}, \ldots, v_{l}=1}^{n} \sum_{\alpha_{1}, \ldots, \alpha_{l}=1}^{m} \int d x_{1} \cdots \int d x_{l} \\
& \quad \times K_{\alpha_{l} \alpha_{1}}^{\left(v_{l} v_{1}\right)} d R_{\alpha_{1}}^{\left(v_{1}\right)}\left(x_{l}, x_{1}\right) K_{\alpha_{1} \alpha_{2}}^{\left(v_{1} v_{2}\right)} R_{\alpha_{2}}^{\left(v_{2}\right)}\left(x_{1}, x_{2}\right) \cdots K_{\alpha_{l}-1 \alpha_{l}}^{\left(v_{l}-1 v_{l}\right)} R_{\alpha_{l}}^{\left(v_{l}\right)}\left(x_{l-1}, x_{l}\right) .
\end{aligned}
$$

Here $d$ denotes the exterior differentiation with respect to the deformation parameters $a_{\mu}, t_{-j, \alpha}^{(\mu)}\left(\mu=1, \ldots, \alpha ; j=1, \ldots, r_{\mu} ; \alpha=1, \ldots, m\right)$. We can show the convergence of this Neumann series by a similar argument as in Section 2.

In [9] a closed 1-form $\omega$ was introduced and the $\tau$ function was defined by $\omega=d \log \tau$. Now we have

\section{Theorem 5.1.}

$$
\omega=d \log \left\langle\varphi_{1}^{(1)} \cdots \varphi_{m}^{(n)}\right\rangle .
$$

Proof. We use the following characterization of $\tau$ function (see (27) in [20] III).

Lemma 5.2. Let $f$ be a function of the deformation parameters. If $f$ satisfies 


$$
\frac{\mathscr{T}_{\alpha}^{(\mu)}(\rho) f}{f} \equiv \sum_{l=0}^{\infty} G_{\alpha \alpha}^{(\mu \mu)(1 l)} \rho^{l} \bmod \rho^{r_{\mu}+1},
$$

then we have $\partial f / \partial t_{-j, \alpha}^{(\mu)}=\partial \tau / \partial t_{-j, \alpha}^{(\mu)}\left(j=1, \ldots, r_{\mu}\right)$.

Together with (3.44) and (4.8), Lemma 5.2 shows

$$
\frac{\partial}{\partial t_{-j, \alpha}^{(\mu)}} \log \tau=\frac{\partial}{\partial t_{-j, \alpha}^{(\mu)}} \log \left\langle\varphi_{1}^{(1)} \cdots \varphi_{m}^{(n)}\right\rangle .
$$

Using the notation of [9] Section 5, we have

$$
\begin{aligned}
& \frac{\partial}{\partial a_{\mu}} \log \tau=\sum_{\alpha=1}^{m} \sum_{j=0}^{r_{\mu}}(j+1) t_{-j, \alpha}^{(\mu)} Z_{j+1 \alpha \alpha}^{(\mu)}, \\
& \frac{\partial}{\partial t_{-j, \alpha}^{(\mu)}} \log \tau=Z_{j, \alpha \alpha}^{(\mu)} .
\end{aligned}
$$

We note also that

$$
\sum_{j=0}^{s-1} G_{\alpha \alpha}^{(\mu \mu)(s-j, j+1)}=s Z_{s, \alpha \alpha}^{(\mu)} .
$$

Hence from (3.37), (3.38), (4.8), (5.6)-(5.9), we have

$$
\begin{aligned}
\frac{\partial}{\partial a_{\mu}} \log \tau & =2 \pi i \sum_{\alpha=1}^{m} \sum_{j=0}^{r_{\mu}} \sum_{k=0}^{j} t_{-j, \alpha}^{(\mu)} \frac{\left\langle\varphi_{1}^{(1)} \cdots \varphi_{\alpha, k-j-1 k+1}^{(\mu)} \cdots \varphi_{m}^{(n)}\right\rangle}{\left\langle\varphi_{1}^{(1)} \cdots \varphi_{m}^{(n)}\right\rangle} \\
& =\frac{\partial}{\partial a_{\mu}} \log \left\langle\varphi_{1}^{(1)} \cdots \varphi_{m}^{(n)}\right\rangle .
\end{aligned}
$$

\section{References}

[1] Sato, M., Miwa, T. and Jimbo, M., Publ. RIMS, Kyoto Univ., 14 (1977), 223 ; 15 (1979), 201, 577, 871; 16 (1980), 531.

[2] Jimbo, M., Miwa, T., Mori, Y. and Sato, M., Physica, 1D (1980), 80.

[3] Jimbo, M. and Miwa, T., Proc. Japan Acad., 56A (1980), 405.

[4] Wu, T. T., McCoy, B. M., Tracy, C. A. and Barouch, E., Phys. Rev., B13 (1976), 316. See also, McCoy, B. M., Tracy, C. A., and Wu, T. T., J. Math. Phys., 18 (1977), 1058: Phys. Lett., 61A (1977), 283.

[5] Schlesinger, L., J. Reine n. Angewandte Math., 141 (1912), 96.

[6] Ueno, K., Master's Thesis, RIMS, Kyoto Univ., 1978.

[ 7 ] — Proc. Japan Acad., 56A (1980), 97, 103, 210.

[8] Flaschka, H. and Newell, A. C., Comm. Math. Phys., 76 (1980), 67.

[9] Jimbo, M., Miwa, T. and Ueno, K., Physica, 2D (1981), 306.

[10] Jimbo, M. and Miwa, T., Proc. Japan Acad., 56A (1980), 143, 149, 269, Physica, 2D (1981) 407. See also, Monodromy preserving deformation of linear ordinary differential equations with rational coefficients, III, RIMS, preprint 344.

[11] Miwa, T., Publ. RIMS, Kyoto Univ., 17 (1981), 703.

[12] — Proc. Japan Acad., 56A (1980), 301. 